Pacific

Journal of

Mathematics

\title{
DOUBLE AFFINE LIE ALGEBRAS AND FINITE GROUPS
}

Nicolas Guay, David Hernandez and Sergey LoKtev 


\title{
DOUBLE AFFINE LIE ALGEBRAS AND FINITE GROUPS
}

\author{
Nicolas Guay, David Hernandez and Sergey Loktev
}

\begin{abstract}
We begin to study the Lie theoretical analogues of symplectic reflection algebras for a finite cyclic group $\Gamma$; we call these algebras "cyclic double affine Lie algebras". We focus on type $A$ : In the finite (respectively affine, double affine) case, we prove that these structures are finite (respectively affine, toroidal) type Lie algebras, but the gradings differ. The case that is essentially new is $\mathfrak{s l}_{n}(\mathbb{C}[u, v] \rtimes \Gamma)$. We describe its universal central extensions and start the study of its representation theory, in particular of its highest weight integrable modules and Weyl modules. We also consider the first Weyl algebra $A_{1}$ instead of the polynomial ring $\mathbb{C}[u, v]$, and, more generally, a rank one rational Cherednik algebra. We study quasifinite highest weight representations of these Lie algebras.
\end{abstract}

1. Introduction 1

2. Matrix Lie algebras over rings 4

3. Cyclic affine Lie algebras 5

4. Cyclic double affine Lie algebras 10

5. Representations of cyclic double affine Lie algebras 15

6. Matrix Lie algebras over rational Cherednik algebras of rank one 24

7. Highest weight representations for matrix Lie algebras over Cherednik algebras of rank one $\quad 30$

8. Further discussions 36

Acknowledgments $\quad 38$

$\begin{array}{ll}\text { References } & 38\end{array}$

\section{Introduction}

Double affine Hecke algebras have been well studied for more than fifteen years now, although they are still very mysterious, and symplectic reflection algebras appeared over seven years ago [Etingof and Ginzburg 2002] as generalizations of

MSC2000: 17B67.

Keywords: affine Lie algebras, toroidal Lie algebras, symplectic reflection algebras.

Hernandez is supported partially by ANR through project "Géométrie et Structures Algébriques Quantiques”. 
double affine Hecke algebras of rational type. Even more mysterious are the double affine Lie algebras and their quantized version introduced in [Ginzburg et al. 1995] and studied for instance in [Hernandez 2005; 2007; Nagao 2007; Nakajima 2002; Schiffmann 2006; Varagnolo and Vasserot 1996; 1998] and the references in the survey [Hernandez 2009].

In this paper, we study candidates for Lie theoretical analogues of symplectic reflection algebras, which we call "cyclic double affine Lie algebras". We look at a family of Lie algebras which have a lot of similarities with affine and double affine Lie algebras, but whose structure depends on a finite cyclic group $\Gamma$.

More precisely, we will be interested in the Lie algebras

$$
\begin{array}{lll}
\mathfrak{s l}_{n}(\mathbb{C}[u] \rtimes \Gamma), & \mathfrak{s l}_{n}(\mathbb{C}[u, v] \rtimes \Gamma), & \mathfrak{s l}_{n}\left(\mathbb{C}\left[u^{ \pm 1}, v\right] \rtimes \Gamma\right), \\
\mathfrak{s l}_{n}\left(\mathbb{C}\left[u^{ \pm 1}\right] \rtimes \Gamma\right), & \mathfrak{s l}_{n}\left(\mathbb{C}[u, v]^{\Gamma}\right), & \mathfrak{s l}_{n}\left(\mathbb{C}\left[u^{ \pm 1}, v^{ \pm 1}\right] \rtimes \Gamma\right),
\end{array}
$$

$\mathfrak{s l}_{n}\left(A_{1} \rtimes \Gamma\right) \quad$ (where $A_{1}$ is the first Weyl algebra), $\mathfrak{s l}_{n}\left(\mathrm{H}_{t, \mathbf{c}}(\Gamma)\right)$ (where $\mathrm{H}_{t, \mathbf{c}}(\Gamma)$ is a rank-one rational Cherednik algebra) and their universal central extensions. This is motivated by the recent work [Guay 2009b], in which deformations of the enveloping algebras of some Lie algebras closely related to these were constructed and connected to symplectic reflection algebras for wreath products via a functor of Schur-Weyl type. When $\Gamma$ is trivial, such deformations in the case of $\mathbb{C}\left[u^{ \pm 1}\right]$ are the affine quantum groups, whereas the case $\mathbb{C}[u]$ corresponds to Yangians. In the double affine setup, the quantum algebras attached to $\mathbb{C}\left[u^{ \pm 1}, v^{ \pm 1}\right], \mathbb{C}\left[u^{ \pm 1}, v\right]$ and $\mathbb{C}[u, v]$ for $\mathfrak{s l}_{n}$ are the quantum toroidal algebras, the affine Yangians and the deformed double current algebras [Guay 2005; 2007].

In this article, we want to study more the structure and representation theory for the Lie algebras above, hoping that, in a future work, we will be able to extend some of our results to the deformed setup. We consider the central extensions for a number of reasons. In the affine case, the full extent of the representation theory comes to life when the center acts not necessarily trivially. Certain presentations of those Lie algebras are actually simpler to state for central extensions since they involve fewer relations; for example, some of the results can be extended without much difficulty to those central extensions. As vector spaces, the centers of the universal extensions are given by certain first cyclic homology groups.

At first sight, one may be tempted to think that introducing the group $\Gamma$ leads to Lie algebras that are different from those that have interested Lie theorists since the advent of Kac-Moody Lie algebras (it was our first motivation), but this is not entirely the case. Indeed, in the one variable case, when we consider not only ordinary polynomials but Laurent polynomials, we prove that we get back affine Lie algebras (Proposition 3.8); this agrees with conjectures of V. Kac [1968] and the classification obtained by V. Kac [1968; 1990] and O. Mathieu [1986; 1992]. 
In the case of Laurent polynomials in two variables, we recover toroidal Lie algebras (Proposition 4.1). (The mixed case $\mathbb{C}\left[u^{ \pm 1}, v\right]$ also does not yield new Lie algebras.) However, when we consider only polynomials in nonnegative powers of the variables, we obtain distinctly new Lie algebras (see Proposition 5.3).

Another motivation comes from geometry. The loop algebra $\mathfrak{s l}_{n}\left(\mathbb{C}\left[u^{ \pm 1}\right]\right)$ can be viewed as the space of polynomial maps $\mathbb{C}^{\times} \rightarrow \mathfrak{s l}_{n}$. One can also consider the affine line instead of the torus $\mathbb{C}^{\times}$, or, more generally, the space of regular maps $X \rightarrow \mathfrak{s l}_{n}$, where $X$ is an arbitrary affine algebraic variety [Feigin and Loktev 2004]. When $X$ is two-dimensional, the most natural candidate is the torus $\mathbb{C}^{\times} \times \mathbb{C}^{\times}$, although a simpler case is the plane $\mathbb{C}^{2}$. The variety $X$ does not necessarily have to be smooth and one interesting singular two-dimensional case is provided by the Kleinian singularities $\mathbb{C}^{2} / G$, where $G$ is a finite subgroup of $\mathrm{SL}_{2}(\mathbb{C})$. We are thus led to the problem of studying the Lie algebras $\mathfrak{s l}_{n}\left(\mathbb{C}[u, v]^{G}\right)$, where $\mathbb{C}[u, v]^{G}$ is the ring of invariant elements for the action of $G$. However, following one of the main ideas explained in the introduction of [Etingof and Ginzburg 2002], it may be interesting to replace $\mathbb{C}[u, v]^{G}$ by the smash product $\mathbb{C}[u, v] \rtimes G$. Moreover, we can expect the full representation theory to come to life when we consider the universal central extensions of $\mathfrak{s l}_{n}\left(\mathbb{C}[u, v]^{G}\right)$ and of $\mathfrak{s l}_{n}(\mathbb{C}[u, v] \rtimes G)$. Feigin and Loktev [2004] showed in the case of a smooth affine variety $X$ that the dimension of the local Weyl modules at a point $p$ does not depend on $p$. One goal is to understand Weyl modules supported at a Kleinian singularity.

This paper is organized as follows. We will denote by $\Gamma$ the group $\mathbb{Z} / d \mathbb{Z}$, whereas $G$ will be a more general finite group. After general reminders on matrix Lie algebra over rings (in particular with the example of $\mathfrak{s l}_{n}(\mathbb{C}[G]$ ) in Section 2, we start with the affine case in Section 3. We study the structure of $\mathfrak{s l}_{n}(\mathbb{C}[u] \rtimes \Gamma)$ and $\mathfrak{s l}_{n}\left(\mathbb{C}\left[u^{ \pm 1}\right] \rtimes \Gamma\right)$, obtain different types of decomposition, and give presentations in terms of generators and relations. We prove that $\mathfrak{s l}_{n}\left(\mathbb{C}\left[u^{ \pm 1}\right] \rtimes \Gamma\right)$ is simply the usual loop algebra $\mathfrak{s l}_{n d}\left(\mathbb{C}\left[t^{ \pm 1}\right]\right)$, but with a nonstandard grading. Guided by the affine setup, we prove analogous results for the double affine cases in Section 4, the representations being studied in Section 5. We consider certain highest weight modules for

$$
\mathfrak{s l}_{n}(\mathbb{C}[u, v] \rtimes \Gamma), \quad \mathfrak{s l}_{n}\left(\mathbb{C}\left[u^{ \pm 1}, v\right] \rtimes \Gamma\right), \quad \mathfrak{s l}_{n}\left(\mathbb{C}\left[u^{ \pm 1}, v^{ \pm 1}\right] \rtimes \Gamma\right)
$$

and state a criterion for the integrability of their unique irreducible quotients. We also study some Weyl modules for $\mathfrak{s l}_{n}(\mathbb{C}[u, v] \rtimes G)$ and $\mathfrak{s l}_{n}\left(\mathbb{C}[u, v]^{\Gamma}\right)$. In the first case, we show that, contrary to what might be expected at first sight, Weyl modules are rather trivial; in the second case, we can apply results of Feigin and Loktev to derive formulas for the dimension some of the local Weyl modules, and we establish a lower bound on their dimensions. In Section 6, assuming usually that $t \neq 0$, we study parabolic subalgebras of $\mathfrak{g l}_{n}\left(\mathrm{H}_{t, \mathbf{c}}(\Gamma)\right)$ and construct an embedding 
of this Lie algebra into a Lie algebra of infinite matrices. This is useful in Section 7 for constructing quasifinite highest weight modules. The main result of this section is a criterion for the quasifiniteness of the irreducible quotients of Verma modules. Further possible directions of research are discussed in Section 8.

\section{Matrix Lie algebras over rings}

2A. General results. In this section, we present general definitions and results that will be useful later. All algebras and tensor products are over $\mathbb{C}$ unless specified otherwise.

Definition 2.1. Let $A$ be an arbitrary associative algebra. The Lie algebra $\mathfrak{s l}_{n}(A)$ is defined as the derived Lie algebra $\left[\mathfrak{g l}_{n}(A), \mathfrak{g l}_{n}(A)\right]$, where $\mathfrak{g l}_{n}(A)=\mathfrak{g l}_{n} \otimes A$.

In other words, the Lie subalgebra $\mathfrak{s l}_{n}(A) \subset \mathfrak{g l}_{n}(A)$ is the sum of $\mathfrak{s l}_{n}(\mathbb{C}) \otimes A$ and of the space of all scalar matrices with entries in $[A, A]$. Thus the cyclic homology group $H C_{0}(A)=A /[A, A]$ accounts for the discrepancy between $\mathfrak{s l}_{n}(A)$ and $\mathfrak{g l}_{n}(A)$.

Since $\mathfrak{s l}_{n}(A)$ is a perfect Lie algebra (that is, $\left[\mathfrak{s l}_{n}(A), \mathfrak{s l}_{n}(A)\right]=\mathfrak{s l}_{n}(A)$ ), it has a universal central extension $\widehat{\mathfrak{s l}}_{n}(A)$ unique up to isomorphism. The following theorem gives a simple presentation of $\widehat{\mathfrak{s l}}_{n}(A)$ in terms of generators and relations.

Theorem 2.2 [Kassel and Loday 1982]. If $n \geq 3$, then $\widehat{\mathfrak{s l}}_{n}(A)$ is isomorphic to the Lie algebra generated by elements $F_{i j}(a)$, for $1 \leq i, j \leq n$ and $a \in A$, that satisfy the relations

$$
\begin{aligned}
{\left[F_{i j}\left(a_{1}\right), F_{j k}\left(a_{2}\right)\right] } & =F_{i k}\left(a_{1} a_{2}\right) & & \text { for } i \neq j \neq k \neq i, \\
{\left[F_{i j}\left(a_{1}\right) F_{k l}\left(a_{2}\right)\right] } & =0, & & \text { for } i \neq j \neq k \neq l \neq i .
\end{aligned}
$$

Here $i \neq j \neq k \neq i$ means that $i \neq j$ and $j \neq k$ and $k \neq i$. We will use this convention in this paper.

When $n=2$, one has to add generators $H_{12}\left(a_{1}, a_{2}\right)$ given by $H_{12}\left(a_{1}, a_{2}\right)=$ [ $\left.F_{12}\left(a_{1}\right), F_{21}\left(a_{2}\right)\right]$ for $a_{1}, a_{2} \in A$, and the relations

$$
\begin{aligned}
& {\left[H_{12}\left(a_{1}, a_{2}\right), F_{12}\left(a_{3}\right)\right]=F_{12}\left(a_{1} a_{2} a_{3}+a_{3} a_{2} a_{1}\right),} \\
& {\left[H_{12}\left(a_{1}, a_{2}\right), F_{21}\left(a_{3}\right)\right]=-F_{21}\left(a_{3} a_{1} a_{2}+a_{2} a_{1} a_{3}\right) .}
\end{aligned}
$$

Kassel and Loday [1982] also prove that the center of $\widehat{\mathfrak{s l}}_{n}(A)$ is isomorphic, as a vector space, to the first cyclic homology group $H C_{1}(A)$. For $G$ a finite group and $A=\mathbb{C}[G]$, we have $H C_{1}(A)=0$, but in the double affine case below, the center will be infinite-dimensional.

The following formulas taken from [Varagnolo and Vasserot 1998] help one to understand better the bracket on $\widehat{\mathfrak{s l}}_{n}(A)$. It is generally difficult to compute explicitly the bracket with respect to the decomposition $\widehat{\mathfrak{s l}}_{n}(A) \cong \mathfrak{s l}_{n}(A) \oplus H C_{1}(A)$, 
but on can get some nice formulas by using a different splitting of $\widehat{\mathfrak{s l}}_{n}(A)$. Let $\langle A, A\rangle$ be the quotient of $A \otimes A$ by the two-sided ideal generated by $a_{1} \otimes a_{2}-a_{2} \otimes a_{1}$ and $a_{1} a_{2} \otimes a_{3}-a_{1} \otimes a_{2} a_{3}-a_{2} \otimes a_{3} a_{1}$. The first cyclic homology group $H C_{1}(A)$ is, by definition, the kernel of the map $\langle A, A\rangle \rightarrow[A, A], a_{1} \otimes a_{2} \mapsto\left[a_{1}, a_{2}\right]$.

For $m_{1}, m_{2} \in \mathfrak{s l}_{n}, a_{1}, a_{2} \in A$, and $(\cdot, \cdot)$ the Killing form on $\mathfrak{s l}_{n}$, set

$$
\left[m_{1}, m_{2}\right]_{+}=m_{1} m_{2}+m_{2} m_{1}-\frac{2}{n}\left(m_{1}, m_{2}\right) I \quad \text { and } \quad\left[a_{1}, a_{2}\right]_{+}=a_{1} a_{2}+a_{2} a_{1} .
$$

Proposition 2.3 [Varagnolo and Vasserot 1998]. The Lie algebra $\widehat{\mathfrak{s l}}_{n}(A)$ is isomorphic to the vector space $\mathfrak{s l}_{n} \otimes A \oplus\langle A, A\rangle$ endowed with the bracket

$$
\begin{aligned}
{\left[m_{1} \otimes a_{1}, m_{2} \otimes a_{2}\right] } & =\frac{1}{n}\left(m_{1}, m_{2}\right)\left\langle a_{1}, a_{2}\right\rangle+\frac{1}{2}\left[m_{1}, m_{2}\right] \otimes\left[a_{1}, a_{2}\right]_{+} \\
{\left[\left\langle a_{1}, a_{2}\right\rangle,\left\langle b_{1}, b_{2}\right\rangle\right] } & =\left\langle\left[a_{1}, a_{2}\right],\left[b_{1}, b_{2}\right]\right\rangle, \\
{\left[\left\langle a_{1}, a_{2}\right\rangle, m_{1} \otimes a_{3}\right] } & =m_{1} \otimes\left[\left[a_{1}, a_{2}\right], a_{3}\right] .
\end{aligned}
$$

2B. Example: Special linear Lie algebras over group rings. Let $G$ be a finite group. One interesting case for us is the group algebra $A=\mathbb{C}[G]$, in which case $H C_{0}(A) \cong \mathbb{C}^{\oplus \operatorname{cl}(G)}$, where $\operatorname{cl}(G)$ is the number of conjugacy classes of $G$.

Lemma 2.4. The Lie algebra $\mathfrak{s l}_{n}(\mathbb{C}[G])$ is semisimple of Dynkin type A.

Proof. Recall that $\operatorname{cl}(G)$ is also the number of irreducible representations of $G$. Enumerate the irreducible representations of $G$ by $\rho_{1}, \ldots, \rho_{\operatorname{cl}(G)}$, and let $d(j)$ be the dimension of $\rho_{j}$. Wedderburn's theorem says that, as algebras, $\mathbb{C}[G]$ is isomorphic to $\bigoplus_{j=1}^{\mathrm{cl}(G)} M_{d(j)}$, where $M_{d(j)}$ is the associative algebra of $d(j) \times d(j)$ matrices. We conclude that $\mathfrak{s l}_{n}(\mathbb{C}[G]) \cong \bigoplus_{j=1}^{\mathrm{cl}(G)} \mathfrak{s l}_{n d(j)}$.

Remark 2.5. The direct sum above is a direct sum of Lie algebras, that is, two different copies of $\mathfrak{s l}_{n}$ commute. A nondegenerate symmetric invariant bilinear form $\kappa$ on the semisimple Lie algebra $\mathfrak{s l}_{n}(\mathbb{C}[G])$ is given by the formula

$$
\kappa\left(m_{1} \gamma_{1}, m_{2} \gamma_{2}\right)=\operatorname{Tr}\left(m_{1} \cdot m_{2}\right) \delta_{\gamma_{1}=\gamma_{2}^{-1}} \quad \text { for } m_{1}, m_{2} \in \mathfrak{s l}_{n} \text { and } \gamma_{1}, \gamma_{2} \in G \text {. }
$$

\section{Cyclic affine Lie algebras}

For an arbitrary ring $R$ with action of a finite group $G$, the ring $R \rtimes G$ is the span of elements $a g$ for $a \in R$ and $g \in G$ with the relations $\left(a_{1} g_{1}\right) \cdot\left(a_{2} g_{2}\right)=a_{1} g_{1}\left(a_{2}\right) g_{1} g_{2}$. In the previous section, just by considering group rings (over $\mathbb{C}$ ), we ended up with semisimple Lie algebras. Here, when $R$ is a Laurent polynomial ring, one can expect to obtain affine Kac-Moody algebras, which is indeed what happens.

3A. Definition and decomposition. Let $\xi$ be a generator of $\Gamma$ and $\zeta$ a primitive $d$ th root of unity. Let $A=\mathbb{C}\left[u^{ \pm 1}\right] \rtimes \Gamma$ and $B=\mathbb{C}[u] \rtimes \Gamma$. The action of $\Gamma$ is defined by $\xi(u)=\zeta u$. We will be interested in the structure of the Lie algebra $\mathfrak{s l}_{n}(A)$ 
and of its universal central extension $\widehat{\mathfrak{s l}}_{n}(A)$. We will also say a few words about $\mathfrak{s l}_{n}(B)$. We will show that $\mathfrak{s l}_{n}(A)$ is a graded simple Lie algebra and explain how it is related to the classification of such Lie algebras obtained by V. Kac [1968; 1990] and O. Mathieu [1986; 1992].

In the following, $\equiv$ is the equivalence modulo $d$.

Lemma 3.1. $\begin{aligned} {[A, A] } & =\bigoplus_{i=1}^{d-1} \mathbb{C}\left[u^{ \pm 1}\right] \xi^{i} \oplus \bigoplus_{j \in \mathbb{Z}, j \neq 0} \mathbb{C} \cdot u^{j}, \\ {[B, B] } & =\bigoplus_{i=1}^{d-1} u \mathbb{C}[u] \xi^{i} \oplus \bigoplus_{j \mathbb{Z}_{\geq 1}, j \neq 0} \mathbb{C} \cdot u^{j} .\end{aligned}$

Proof. If $1 \leq i \leq d-1$, then $u^{j} \xi^{i}=\left[u, u^{j-1} \xi^{i}\right] /\left(1-\zeta^{i}\right)$. If $j \in \mathbb{Z}$ and $j \not \equiv 0$, then $u^{j}=\left[\xi, u^{j} \xi^{-1}\right] /\left(\zeta^{j}-1\right)$. This proves $\supseteq$. Consider

$$
\left[u^{k} \xi^{a}, u^{m} \xi^{b}\right]=\left(\zeta^{a m}-\zeta^{b k}\right) u^{k+m} \xi^{a+b}
$$

and suppose that the right-hand side is in $\mathbb{C}\left[u^{ \pm 1}\right]^{\Gamma}$. Then $k+l \equiv 0$ and $a+b \equiv 0$, so $\zeta^{a m}-\zeta^{b k}=0$. This proves $\subseteq$.

Corollary 3.2. $H C_{0}(B) \cong \mathbb{C}[u]^{\Gamma} \oplus \mathbb{C}[\Gamma]$.

The Lie algebra $\mathfrak{s l}_{n}(A)$ admits different vector space decompositions, similar to the two standard triangular decompositions of affine Lie algebras. Let $\mathfrak{n}^{+}$(respectively $\mathfrak{n}^{-}$) be the Lie algebra of strictly upper (respectively lower) triangular matrices in $\mathfrak{s l}_{n}$ (over $\mathbb{C}$ ), and let $\mathfrak{h}$ be the usual Cartan subalgebra of $\mathfrak{s l}_{n}$. The elementary matrices in $\mathfrak{g l}_{n}$ will be denoted $E_{i j}$ and $I$ will stand for the identity matrix. In the following, by abuse of notation, an element $g \otimes a$ will be denoted $g a$ for $g \in \mathfrak{g l}_{n}$ and $a \in A$. We have the vector space isomorphisms (triangular decompositions)

$$
\mathfrak{s l}_{n}(A) \cong\left(\mathfrak{n}^{-} A\right) \oplus(\mathfrak{h} A \oplus I[A, A]) \oplus\left(\mathfrak{n}^{+} A\right),
$$

and $\mathfrak{s l}_{n}(A)$ is also isomorphic to the sum

$$
\begin{aligned}
\left(\mathfrak{s l}_{n} u^{-1} \mathbb{C}\left[u^{-1}\right] \rtimes \Gamma \oplus\left(\bigoplus_{\substack{j \leq-1, j \neq 0 \\
0 \leq i \leq d-1}} \mathbb{C} I u^{j} \xi^{i} \oplus \bigoplus_{1 \leq i \leq d-1} I u^{-d} \mathbb{C}\left[u^{-d}\right] \xi^{i}\right) \oplus \mathfrak{n}^{-} \mathbb{C}[\Gamma]\right) \\
\\
\oplus\left(\mathfrak{h} \mathbb{C}[\Gamma] \oplus \bigoplus_{1 \leq i \leq d-1} \mathbb{C} I \xi^{i}\right) \\
\oplus\left(\mathfrak{s l}_{n} u \mathbb{C}[u] \rtimes \Gamma \oplus\left(\bigoplus_{\substack{j \geq 1, j \neq 0 \\
0 \leq i \leq d-1}} \mathbb{C} I u^{j} \xi^{i} \oplus \bigoplus_{1 \leq i \leq d-1} I u^{d} \mathbb{C}\left[u^{d}\right] \xi^{i}\right) \oplus \mathfrak{n}^{+} \mathbb{C}[\Gamma]\right) .
\end{aligned}
$$


These lead to similar decompositions for $\mathfrak{s l}_{n}(B)$. These triangular decompositions are similar to those considered, for instance, in [Khare 2009].

The first triangular decomposition is analogous to the loop triangular decomposition of affine Lie algebras, but in our situation the middle Lie algebra is not commutative. The second triangular decomposition is similar to the decomposition of affine Lie algebras adapted to Chevalley-Kac generators, and it is of particular importance as the middle term $\mathfrak{H}$ in commutative. So the role of the Cartan subalgebra will be played by the commutative Lie algebra $\mathfrak{H}$ and our immediate aim is to obtain a corresponding appropriate root space decomposition of $\mathfrak{s l}_{n}(A)$.

It will be convenient to work with the primitive idempotents of $\Gamma$, so let us set $\mathbf{e}_{j}=\frac{1}{d} \sum_{i=0}^{d-1} \zeta^{-i j} \xi^{i}$. A vector space basis of $\mathfrak{H}$ is given by

$$
H_{i, j}= \begin{cases}\left(E_{i, i}-E_{i+1, i+1}\right) \mathbf{e}_{j} & \text { for } 1 \leq i \leq n-1 \text { and } 0 \leq j \leq d-1, \\ E_{n, n} \mathbf{e}_{j}-E_{1,1} \mathbf{e}_{j+1} & \text { for } i=0 \text { and } 0 \leq j \leq d-2 .\end{cases}
$$

We note that we could define $H_{0, d-1}$ in the same way, but then we would get $\sum_{i=0}^{n-1} \sum_{j=0}^{d-1} H_{i, j}=0$ in $\mathfrak{s l}_{n}(A)$ (but lifts to a nonzero central element in $\widehat{\mathfrak{s l}}_{n}(A)$ ).

Lemma 3.3. A basis of the eigenspaces for nonzero eigenvalues for the adjoint action of $\mathfrak{H}$ on $\mathfrak{s l}_{n}(A)$ (except for $\mathfrak{H}$ itself) is given by the vectors

$$
\begin{aligned}
& E_{i j} u^{k} \mathbf{e}_{l} \quad \text { for } 1 \leq i \neq j \leq n, \quad k \in \mathbb{Z}, \quad 0 \leq l \leq d-1, \\
& E_{i i} u^{k} \mathbf{e}_{l} \quad \text { for } 1 \leq i \leq n, \quad k \neq 0, \quad 0 \leq l \leq d-1 .
\end{aligned}
$$

Proof. This is a consequence of some simple computations: For

$$
1 \leq i \neq j \leq n, \quad 0 \leq a \leq n-1, \quad k \in \mathbb{Z}, \quad 0 \leq l \leq d-1, \quad 0 \leq b \leq d-1,
$$

we have

$\left[H_{a, b}, E_{i j} u^{k} \mathbf{e}_{l}\right]=\left\{\begin{array}{r}\left(\delta_{b \equiv l}\left(\delta_{a+1 \equiv j}-\delta_{a \equiv j}\right)+\delta_{b-k \equiv l}\left(\delta_{a \equiv i}-\delta_{a+1 \equiv i}\right)\right) E_{i j} u^{k} \mathbf{e}_{l} \\ \text { if } a \neq 0 \\ \left(\delta_{n \equiv i} \delta_{b-k \equiv l}-\delta_{n \equiv j} \delta_{b \equiv l}-\delta_{1 \equiv i} \delta_{b+1-k \equiv l}-\delta_{j \equiv 1} \delta_{b+1 \equiv l}\right) E_{i j} u^{k} \mathbf{e}_{l} \\ \text { if } a=0 .\end{array}\right.$

For $1 \leq i \leq n, k \neq \equiv 0$ and $0 \leq l \leq d-1$, we have

$$
\left[H_{a, b}, E_{i i} u^{k} \mathbf{e}_{l}\right]=\left\{\begin{array}{lr}
\left(\delta_{a \equiv i}-\delta_{a+1 \equiv i}\right)\left(\delta_{b-k \equiv l}-\delta_{b \equiv l}\right) E_{i i} u^{k} \mathbf{e}_{l} & \text { if } a \neq 0, \\
\left(\delta_{n \equiv i}\left(\delta_{b-k \equiv l}-\delta_{b \equiv l}\right)-\delta_{i \equiv 1}\left(\delta_{b+1-k \equiv l}-\delta_{b+1 \equiv l}\right)\right) E_{i i} u^{k} \mathbf{e}_{l} & \text { if } a=0,
\end{array}\right.
$$

which proves the lemma. 
3B. Derivation element and imaginary roots. We will introduce the real roots, imaginary roots, and root spaces after adding a derivation $\mathrm{d}$ to $\widehat{\mathfrak{s l}}_{n}(A)$.

The center of the universal central extension $\widehat{\mathfrak{s l}}_{n}(A)$ of $\mathfrak{s l}_{n}(A)$ is isomorphic to $H C_{1}(A)$; see [Kassel and Loday 1982]. It is known that $H C_{1}(A)$ is isomorphic to $\left(\mathbb{C}\left[u^{ \pm 1}\right] d u\right)^{\Gamma} / d\left(\mathbb{C}\left[u^{ \pm 1}\right]^{\Gamma}\right)$, that is, the quotient of the space of $\Gamma$-invariant 1 -forms on $\mathbb{C}^{\times}$by the space of exact 1 -forms coming from $\Gamma$-invariant Laurent polynomials. This can be deduced from the isomorphism $A \cong M_{d}\left(\mathbb{C}\left[t^{ \pm 1}\right]\right)$ - see Proposition 3.8. Thus this cyclic homology group is one-dimensional, with basis given by $u^{-1} d u$, which we denote as usual by c.

Definition 3.4. The cyclic affine Lie algebra $\overline{\mathfrak{s l}}_{n}(A)$ is obtained from $\widehat{\mathfrak{s l}}_{n}(A)$ by adding a derivation $\mathrm{d}$ that satisfies the relations $\left[\mathrm{d}, E_{i j} u^{k} \mathbf{e}_{l}\right]=k E_{i j} u^{k} \mathbf{e}_{l}$.

Set $\overline{\mathfrak{H}}=\mathfrak{H} \oplus \mathbb{C} \cdot c \oplus \mathbb{C} \cdot d$. We can now introduce the roots as appropriate elements of $\overline{\mathfrak{H}}_{0}^{*}=\left\{\lambda \in \overline{\mathfrak{H}}^{*} \mid \lambda(\mathrm{c})=0\right\}$. The real root spaces are spanned by the root vectors $E_{i j} u^{k} \mathbf{e}_{l}$, where $0 \leq l \leq d-1,1 \leq i, j \leq n$ and $k \in \mathbb{Z}$, with the condition that $k \not \equiv 0$ if $i=j$.

The imaginary root spaces are spanned by the root vectors

$$
\begin{array}{lr}
H_{i} u^{k d} \mathbf{e}_{l} \quad \text { for } 1 \leq i \leq n-1, \quad k \neq 0, & 0 \leq l \leq d-1, \\
E_{n n} u^{k d} \mathbf{e}_{l}-E_{11} u^{k d} \mathbf{e}_{l+1} \quad \text { for } k \neq 0, & 0 \leq l \leq d-2 .
\end{array}
$$

We want to identify the root lattice as a lattice in $\overline{\mathfrak{H}}_{0}^{*}$. Let us introduce the elements $\epsilon_{i, l} \in \overline{\mathfrak{H}}_{0}^{*}$ for $1 \leq i \leq n$ and $0 \leq l \leq d-1$ by setting

$$
\epsilon_{i, l}\left(H_{a, b}\right)=\left(\delta_{a=i}-\delta_{a+1=i}\right) \delta_{b \equiv l}, \quad \epsilon_{i, l}\left(H_{0, b}\right)=\delta_{n=i} \delta_{b \equiv l}-\delta_{i=1} \delta_{b+1} \equiv l,
$$

and $\epsilon_{i, l}(\mathrm{~d})=0$.

Definition 3.5. The real roots are $\epsilon_{i, k+l}-\epsilon_{j, l}+k \delta$ for $1 \leq i, j \leq n, 0 \leq l \leq d-1$ and $k \in \mathbb{Z}$ with $i \neq j$ or, if $i=j$, then $k \equiv 0$; the imaginary ones are $k d \delta$, where $\delta(\mathrm{d})=1, \delta\left(H_{a, b}\right)=0$ and $k \neq 0$.

They generate a lattice - the root lattice - in $\overline{\mathfrak{H}}_{0}^{*}$. As one can verify, the real root spaces all have dimension one.

Lemma 3.6. The root lattice is freely generated by the roots

$$
\begin{array}{ll}
\epsilon_{i, l}-\epsilon_{i+1, l} & \text { for } 1 \leq i \leq n-1 \text { and } 0 \leq l \leq d-1, \\
\epsilon_{n, l}-\epsilon_{1, l+1} & \text { for } 0 \leq l \leq d-2, \\
\left(\epsilon_{n, d-1}-\epsilon_{1,0}\right)+\delta ; &
\end{array}
$$

we call these the positive simple roots. 
Proof. Note that

$$
\delta=\left(\left(\epsilon_{n, d-1}-\epsilon_{1,0}\right)+\delta\right)+\sum_{l=0}^{d-1} \sum_{i=1}^{n-1}\left(\epsilon_{i, l}-\epsilon_{i+1, l}\right)+\sum_{l=0}^{d-2}\left(\epsilon_{n, l}-\epsilon_{1, l+1}\right)
$$

The set of simple roots contains $n d$ elements, which is also $\operatorname{dim} \overline{\mathfrak{H}}_{0}^{*}$.

\section{C. Cyclic affine Lie algebras and affine Lie algebras.}

Proposition 3.7. The Lie algebra $\mathfrak{s l}_{n}(A)$ is graded simple (that is, it contains no nontrivial graded ideal).

Proof. Suppose that $\tilde{I}=\sum_{m \in \mathbb{Z}} \tilde{I}_{m}$ is a nonzero graded ideal of $\mathfrak{s l}_{n}\left(\mathbb{C}\left[u^{ \pm 1}\right] \rtimes \Gamma\right)$. Then $\tilde{I}$ is stable under the adjoint action of $\mathfrak{H}$; hence each graded piece $\tilde{I}_{m}$ must decompose into the direct sum of all the root spaces contained in $\tilde{I}_{m}$. It can be checked that the ideal generated by any real root vector is the whole $\mathfrak{s l}_{n}(A)$, so if $\tilde{I}$ contains a real root vector, then $\tilde{I}$ is the whole Lie algebra. Moreover, if $\tilde{I}$ contains an imaginary root vector, then it contains also a real one.

However, the Lie algebra $\mathfrak{s l}_{n}(A)$ is not simple if we do not take the grading into account, as can be seen from Proposition 3.8 below. A conjecture of V. Kac [1968], proved in general by O. Mathieu [1986; 1992], gives a classification of graded simple Lie algebras of polynomial growth according to which, following Proposition 3.7, $\mathfrak{s l}_{n}(A)$ must be isomorphic to a (perhaps twisted) loop algebra. This is indeed the case, although the isomorphism does not respect the natural grading on the loop algebra $\mathfrak{s l}_{n d} \otimes \mathbb{C}\left[t^{ \pm 1}\right]$.

Proposition 3.8. The Lie algebras $\mathfrak{s l}_{n}(A)$ and $\mathfrak{s l}_{n d}\left(\mathbb{C}\left[t^{ \pm 1}\right]\right)$ are isomorphic.

Proof. An isomorphism is given explicitly by the following formulas: If

$$
0 \leq l \leq d-1, \quad k \in \mathbb{Z}, \quad-l \leq r \leq d-l-1, \quad 1 \leq i \neq j \leq n,
$$

then

$$
\begin{aligned}
E_{n(l+r)+i, n l+j} t^{k} & \leftrightarrow E_{i j} u^{k d+r} \mathbf{e}_{l}, & & \\
E_{n(l+r)+i, n l+i} t^{k} & \leftrightarrow E_{i i} u^{k d+r} \mathbf{e}_{l} & & \text { for } r \neq 0, \\
\left(E_{n l+i, n l+i}-E_{n l+i+1, n l+i+1}\right) t^{k} & \leftrightarrow H_{i} u^{k d} \mathbf{e}_{l} & & \text { for } i \neq n, \\
\left(E_{n l, n l}-E_{n l+1, n l+1}\right) t^{k} & \leftrightarrow E_{n n} u^{k d} \mathbf{e}_{l}-E_{11} u^{k d} \mathbf{e}_{l+1} & & \text { for } l \neq 0 .
\end{aligned}
$$

These formulas can be obtained via the algebra isomorphism $A \cong M_{d}\left(\mathbb{C}\left[t^{ \pm 1}\right]\right)$ that is given by $E_{l+r, l} t^{k} \leftrightarrow u^{k d+r} \mathbf{e}_{l}$.

Let $\phi: \mathfrak{s l}_{n d}\left(\mathbb{C}\left[t^{ \pm 1}\right]\right) \stackrel{\sim}{\longrightarrow} \mathfrak{s l}_{n}\left(\mathbb{C}\left[u^{ \pm 1}\right] \rtimes \Gamma\right)$ be the isomorphism in the proof of Proposition 3.8. We can put a grading on $\mathfrak{s l}_{n d}$ by giving $E_{i j}$ degree $j-i$. This induces another grading on $\mathfrak{s l}_{n d}\left(\mathbb{C}\left[t^{ \pm 1}\right]\right)$ besides the one coming from the powers of $t$; the total of these two is the grading given by $\operatorname{deg}\left(E_{i j}\left(t^{r}\right)\right)=j-i+r$. 
Proposition 3.9. $\phi$ is an isomorphism of graded Lie algebras when $\mathfrak{s l}_{n d}\left(\mathbb{C}\left[t^{ \pm 1}\right]\right)$ is endowed with the total grading and $\mathfrak{s l}_{n}\left(\mathbb{C}\left[u^{ \pm 1}\right] \rtimes \Gamma\right)$ is graded by powers of $u$.

Let $\mathfrak{p}$ be the parabolic subalgebra of $\mathfrak{s l}_{n d}$ consisting of all the lower triangular matrices and the matrices with $d$ blocks of $n \times n$ matrices along the diagonal. Let $\mathfrak{n}$ be the nilpotent subalgebra that consists of all the upper triangular matrices except those in these blocks along the diagonal, so that $\mathfrak{s l}_{n d} \cong \mathfrak{p} \oplus \mathfrak{n}$. The isomorphism given in Proposition 3.8 shows that $\mathfrak{s l}_{n}(B)$ is isomorphic to $\mathfrak{p} \otimes \mathbb{C}[t] \oplus \mathfrak{n} \otimes t \mathbb{C}[t]$.

We gave in the previous subsection two triangular decompositions of $\mathfrak{s l}_{n}(A)$. The Lie algebra $\mathfrak{s l}_{n d}\left(\mathbb{C}\left[t^{ \pm 1}\right]\right)$ admits similar decompositions, namely,

$$
\begin{aligned}
& \mathfrak{s l}_{n d}\left(\mathbb{C}\left[t^{ \pm 1}\right]\right) \cong\left(\mathfrak{n}_{n d}^{-} \otimes \mathbb{C}\left[t^{ \pm 1}\right]\right) \oplus\left(\mathfrak{h}_{n d} \otimes \mathbb{C}\left[t^{ \pm 1}\right]\right) \oplus\left(\mathfrak{n}_{n d}^{+} \otimes \mathbb{C}\left[t^{ \pm 1}\right]\right), \\
& \mathfrak{s l}_{n d}\left(\mathbb{C}\left[t^{ \pm 1}\right]\right) \cong\left(\mathfrak{s l}_{n d} \otimes t^{-1} \mathbb{C}\left[t^{-1}\right] \oplus \mathfrak{n}_{n d}^{-}\right) \oplus \mathfrak{h}_{n d} \oplus\left(\mathfrak{n}_{n d}^{+} \oplus \mathfrak{s l} \mathfrak{l}_{n d} \otimes t \mathbb{C}[t]\right) .
\end{aligned}
$$

The isomorphism given in Proposition 3.8 preserves the second decomposition, but not the first one.

We conclude that a lot is already known about the representation theory of cyclic affine algebras, and simple finite-dimensional representations are classified by tuples of $n d-1$ polynomials; see [Chari 1986]. We state this more explicitly for toroidal Lie algebras in Section 5A below.

\section{Cyclic double affine Lie algebras}

In this section, we set $A=\mathbb{C}\left[u^{ \pm 1}, v^{ \pm 1}\right] \rtimes \Gamma, B=\mathbb{C}\left[u^{ \pm 1}, v\right] \rtimes \Gamma, C=\mathbb{C}[u, v] \rtimes \Gamma$. Here, $\xi$ acts on $u$ and $v$ by $\xi(u)=\zeta u$ and $\xi(v)=\zeta^{-1} v$. Note that, setting $w=u v$, we deduce that $A$ is isomorphic to $\mathbb{C}\left[u^{ \pm 1}, w^{ \pm 1}\right] \rtimes \Gamma$, where $\Gamma$ acts trivially on $w$. The same remark applies to $B$.

We will be interested in the structure of the Lie algebras $\mathfrak{s l}_{n}(A), \mathfrak{s l}_{n}(B), \mathfrak{s l}_{n}(C)$ and their universal central extensions.

4A. Structure. We need to know certain cyclic homology groups. For example,

$$
H C_{0}(A)=A^{\Gamma}, \quad H C_{0}(B)=B^{\Gamma}, \quad H C_{0}(C)=C^{\Gamma} \oplus \mathbb{C}^{\oplus(|\Gamma|-1)},
$$

$H C_{1}(A) \cong \Omega^{1}(A)^{\Gamma} / d\left(A^{\Gamma}\right)$, and similarly for $B$ and $C$ (see Corollary 4.3). As vector spaces, when $\Gamma \neq\{\mathrm{id}\}$, we have

$$
\begin{aligned}
H C_{1}(A) & =v \mathbb{C}\left[u^{ \pm 1}, v^{ \pm 1}\right]^{\Gamma} d u \oplus \mathbb{C}\left[u^{ \pm 1}\right]^{\Gamma} v^{-1} d v \\
& =\left(\mathbb{C}\left[u^{ \pm 1}\right]^{\Gamma} \otimes_{\mathbb{C}} \mathbb{C}\left[w^{ \pm 1}\right]\right) u^{-1} d u \oplus \mathbb{C}\left[u^{ \pm 1}\right]^{\Gamma} w^{-1} d w, \\
H C_{1}(B) & =v \mathbb{C}\left[u^{ \pm 1}, v\right]^{\Gamma} d u \oplus \mathbb{C} u^{-1} d u \\
& \cong\left(w \mathbb{C}[w] \otimes_{\mathbb{C}} \mathbb{C}\left[u^{ \pm 1}\right]^{\Gamma}\right) u^{-1} d u \oplus \mathbb{C} u^{-1} d u, \\
H C_{1}(C) & =v \mathbb{C}[u, v]^{\Gamma} d u .
\end{aligned}
$$


These results can be obtained from the computations of Hochschild homology in [Farinati 2005], which are valid for more general groups than $\Gamma$. For $A$ and $B$, they can also be deduced from the proof of this extension of Proposition 3.8:

Proposition 4.1. The Lie algebra $\mathfrak{s l}_{n}(A)$ is isomorphic to the toroidal Lie algebra $\mathfrak{s l}_{n d}\left(\mathbb{C}\left[s^{ \pm 1}, t^{ \pm 1}\right]\right)$; likewise $\mathfrak{s l}_{n}(B)$ is isomorphic to $\mathfrak{s l}_{n d}\left(\mathbb{C}\left[s^{ \pm 1}, t\right]\right)$.

Proof. We write $A=\mathbb{C}\left[u^{ \pm 1}, w^{ \pm 1}\right]$. Since $\mathbb{C}\left[u^{ \pm 1}\right] \rtimes \Gamma \cong M_{d}\left(\mathbb{C}\left[s^{ \pm 1}\right]\right)$ with $s=u^{d}$ (see the proof of Proposition 3.8) and $A \cong\left(\mathbb{C}\left[u^{ \pm}\right] \rtimes \Gamma\right) \otimes \mathbb{C} \mathbb{C}\left[w^{ \pm 1}\right]$, we immediately deduce the first claim by setting $t=w$. The same argument applies to $B$.

Remark 4.2. The isomorphism in this proposition is reminiscent of [Berman et al. 2003, Lemma 4.1]. In that article, the authors used vertex operator techniques to construct representations of a certain affinization of $\mathfrak{g l}_{N}\left(\mathbb{C}[G]\left[t, t^{-1}\right]\right)$, where $G$ is an admissible subgroup of $\mathbb{C}^{\times}$(in the sense defined therein).

Explicitly, the isomorphism in Proposition 4.1 sends $E_{a b} u^{i} w^{j} \mathbf{e}_{k}$ with $i=m+d l$, $-k \leq m \leq d-1-k$ and $0 \leq k \leq d-1$ to $E_{a+(m+k) n, b+k n} s^{l} t^{j}$; in terms of $u, v$ instead of $u, w$, it maps $E_{a b} u^{i} v^{j} \mathbf{e}_{k}$ with $i-j=m+d l$ and $-k \leq m \leq d-$ $1-k$ to $E_{a+(m+k) n, b+k n} s^{l} t^{j}$. In particular, if $i=0$, then this map restricts to the isomorphism of Proposition 3.8 for $\mathbb{C}\left[v^{ \pm 1}\right]$, with $v$ playing the role of $u^{-1}$. The Lie subalgebra $\mathfrak{s l}_{n}\left(\mathbb{C}\left[w^{ \pm 1}\right] \rtimes \Gamma\right)$ gets identified with the direct sum of $d$ copies of $\mathfrak{s l}_{n}\left(\mathbb{C}\left[t^{ \pm 1}\right]\right)$, which agrees with $\mathbb{C}\left[w^{ \pm 1}\right] \rtimes \Gamma \cong \mathbb{C}\left[w^{ \pm 1}\right] \otimes_{\mathbb{C}} \mathbb{C}[\Gamma] \cong \mathbb{C}\left[w^{ \pm 1}\right]^{\oplus d}$.

Corollary 4.3. The cyclic homology groups $H C_{1}(A)$ and $H C_{1}(B)$ are given by

$$
H C_{1}(A) \cong \frac{\Omega^{1}\left(\mathbb{C}\left[s^{ \pm 1}, t^{ \pm 1}\right]\right)}{d\left(\mathbb{C}\left[s^{ \pm 1}, t^{ \pm 1}\right]\right)} \quad \text { and } \quad H C_{1}(B) \cong \frac{\Omega^{1}\left(\mathbb{C}\left[s^{ \pm 1}, t\right]\right)}{d\left(\mathbb{C}\left[s^{ \pm 1}, t\right]\right)}
$$

Proof. This follows from the algebra isomorphism $\left.A \cong M_{d}\left(\mathbb{C}\left[s^{ \pm 1}, t^{ \pm 1}\right]\right)\right)$ in the proof of Proposition 4.1. (A similar isomorphism holds for $B$.)

When we restrict the isomorphism of Proposition 4.1 to $\mathfrak{s l}_{n}(C)$, we obtain an injective map from $\mathfrak{s l}_{n}(C)$ into $\mathfrak{s l}_{n d}(\mathbb{C}[s, t])$. It comes from an injection of $C$ into $M_{d}(\mathbb{C}[s, t])$. This latter map is a special case $(n=1$ and $x, y, v=0)$ of the homomorphism introduced in [Gordon 2007, Section 6.1]; see also [CrawleyBoevey 1991]. It is not an epimorphism from $C$ to $M_{d}(\mathbb{C}[s, t])$ in the set theoretical sense, but it is an epimorphism in the following categorical sense: Any two ring homomorphisms $M_{d}(\mathbb{C}[s, t]) \rightarrow D$ whose composites with $C \hookrightarrow M_{d}(\mathbb{C}[s, t])$ agree on $C$ must be equal.

Using Proposition 4.1 and the explicit isomorphism given right after the proof, we can give a formula for the bracket on $\widehat{\mathfrak{s l}}_{n}(A)$. It is easier to do it first with $u, w$ and then translate the result to $u, v$. Note that we identify the center of $\widehat{\mathfrak{s l}}_{n}(A)$ with $\Omega^{1}\left(\mathbb{C}\left[s^{ \pm 1}, t^{ \pm 1}\right]\right) / d\left(\mathbb{C}\left[s^{ \pm 1}, t^{ \pm 1}\right]\right)$, so that it makes sense to write $d(f)$ for some 
$f \in \mathbb{C}\left[s^{ \pm 1}, t^{ \pm 1}\right]$, with $s=u^{d}$ and $t=w$ as above. For a commutative algebra $R$, the bracket on $\mathfrak{s l}_{n}(R)$ is given by

$$
\left[E_{a_{1} b_{1}} r_{1}, E_{a_{2} b_{2}} r_{2}\right]=\left[E_{a_{1} b_{1}}, E_{a_{2} b_{2}}\right] r_{1} r_{2}+\operatorname{Tr}\left(E_{a_{1} b_{1}} E_{a_{2} b_{2}}\right) r_{1} d\left(r_{2}\right),
$$

where $\operatorname{Tr}$ is the usual trace functional [Kassel 1984] and $r_{1} d\left(r_{2}\right) \in \Omega^{1}(R) / d R$. Now consider

$$
\begin{aligned}
& {\left[E_{a_{1}+\left(m_{1}+k_{1}\right) n, b_{1}+k_{1} n} s^{l_{1}} t^{j_{1}}, E_{a_{2}+\left(m_{2}+k_{2}\right) n, b_{2}+k_{2} n} s^{l_{2}} t^{j_{2}}\right]} \\
& =\delta_{b_{1}+k_{1} n=a_{2}+\left(m_{2}+k_{2}\right) n} E_{a_{1}+\left(m_{1}+k_{1}\right) n, b_{2}+k_{2} n} s^{l_{1}+l_{2}} t^{j_{1}+j_{2}} \\
& \quad-\delta_{b_{2}+k_{2} n=a_{1}+\left(m_{1}+k_{1}\right) n} E_{a_{2}+\left(m_{2}+k_{2}\right) n, b_{1}+k_{1} n} s^{l_{1}+l_{2}} t^{j_{1}+j_{2}} \\
& \quad+\delta_{a_{1}+\left(m_{1}+k_{1}\right) n=b_{2}+k_{2} n} \delta_{b_{1}+k_{1} n=a_{2}+\left(m_{2}+k_{2}\right) n} s^{l_{1}} t^{j_{1}} d\left(s^{l_{2}} t^{j_{2}}\right) .
\end{aligned}
$$

The isomorphism of Proposition 4.1 identifies the left side with

$$
\left[E_{a_{1} b_{1}} u^{i_{1}} w^{j_{1}} \mathbf{e}_{k_{1}}, E_{a_{2} b_{2}} u^{i_{2}} w^{j_{2}} \mathbf{e}_{k_{2}}\right] \quad \text { where } i_{1}=d l_{1}+m_{1} \text { and } i_{2}=d l_{2}+m_{2},
$$

whereas the right side is identified with

$$
\begin{aligned}
& \delta_{a_{2}=b_{1}} \delta_{k_{1} \equiv m_{2}+k_{2}} E_{a_{1}, b_{2}} u^{i_{1}+i_{2}} w^{j_{1}+j_{2}} \\
- & \delta_{b_{2}=a_{1}} \delta_{m_{1}+k_{1} \equiv k_{2}} E_{a_{2} b_{1}} u^{i_{1}+i_{2}} w^{j_{1}+j_{2}} \\
+ & \delta_{a_{1}=b_{2}} \delta_{m_{1}+k_{1} \equiv k_{2}} \delta_{b_{1}=a_{2}} \delta_{k_{1} \equiv m_{2}+k_{2}} u^{i_{1}-m_{1}} w^{j_{1}} d\left(u^{i_{2}-m_{2}} w^{j_{2}}\right) .
\end{aligned}
$$

Setting $v=w u^{-1}$, we obtain a formula in terms of $u$ and $v$ if we now define $m_{1}$ and $m_{2}$ by $i_{i}-j_{i}=m_{i}+l_{i} d$ :

$$
\begin{aligned}
& {\left[E_{a_{1} b_{1}} u^{i_{1}} v^{j_{1}} \mathbf{e}_{k_{1}}, E_{a_{2} b_{2}} u^{i_{2}} v^{j_{2}} \mathbf{e}_{k_{2}}\right]} \\
& =\delta_{a_{2}=b_{1}} \delta_{k_{1} \equiv m_{2}+k_{2}} E_{a_{1}, b_{2}} u^{i_{1}+i_{2}} v^{j_{1}+j_{2}}-\delta_{b_{2}=a_{1}} \delta_{m_{1}+k_{1} \equiv k_{2}} E_{a_{2} b_{1}} u^{i_{1}+i_{2}} v^{j_{1}+j_{2}} \\
& +\delta_{a_{1}=b_{2}} \delta_{m_{1}+k_{1} \equiv k_{2}} \delta_{b_{1}=a_{2}} \delta_{k_{1} \equiv m_{2}+k_{2}} u^{i_{1}-m_{1}} v^{j_{1}} d\left(u^{i_{2}-m_{2}} v^{j_{2}}\right) .
\end{aligned}
$$

The bracket when $B$ and $C$ replace $A$ has exactly the same formula; simply restrict the values allowed for $i_{1}, i_{2}, j_{1}, j_{2}$. Proposition 2.3's description of $\widehat{\mathfrak{s l}}_{n}(A)$ implies that the natural maps $\widehat{\mathfrak{s l}}_{n}(B), \widehat{\mathfrak{s l}}_{n}(C) \rightarrow \widehat{\mathfrak{s l}}_{n}(A)$ are embeddings.

In the next proposition, we use Proposition 4.1 to adapt [Moody et al. 1990, Proposition 3.5] to $\widehat{\mathfrak{s l}}_{n}(A)$. Our Proposition 4.4 has a few more relations, but the proofs are the same.

Let $\mathbf{C}=\left(c_{i j}\right)$ be the affine Cartan matrix of type $\widehat{A}_{n d-1}$ with rows and columns indexed from 0 to $n d-1$. Let $f:[0, n-1] \times[0, d-1] \rightarrow[0, n d-1]$ be the function $f(i, j)=i+j n$.

Proposition 4.4. The Lie algebra $\widehat{\mathfrak{s l}}_{n}(A)$ is isomorphic to the Lie algebra $\mathfrak{t}$ generated by the elements $X_{i, j, r}^{ \pm}$and $H_{i, j, r}$ for $0 \leq i \leq n-1,0 \leq j \leq d-1$ and $r \in \mathbb{Z}$, 
and a central element $\mathrm{c}$ satisfying relations

$$
\begin{aligned}
& {\left[H_{i_{1}, j_{1}, r_{1}}, H_{i_{2}, j_{2}, r_{2}}\right]=r_{1} c_{f\left(i_{1}, j_{1}\right), f\left(i_{2}, j_{2}\right)} \delta_{r_{1}+r_{2}=0} \mathrm{c}} \\
& {\left[H_{i_{1}, j_{1}, 0}, X_{i_{2}, j_{2}, r_{2}}^{ \pm}\right]= \pm c_{f\left(i_{1}, j_{1}\right), f\left(i_{2}, j_{2}\right)} X_{i_{2}, j_{2}, r_{2}}^{ \pm},} \\
& {\left[H_{i_{1}, j_{1}, r_{1}+1}, X_{i_{2}, j_{2}, r_{2}}^{ \pm}\right]=\left[H_{i_{1}, j_{1}, r_{1}}, X_{i_{2}, j_{2}, r_{2}+1}^{ \pm}\right] \text {, }} \\
& {\left[X_{i_{1}, j_{1}, r_{1}+1}^{ \pm}, X_{i_{2}, j_{2}, r_{2}}^{ \pm}\right]=\left[X_{i_{1}, j_{1}, r_{1}}^{ \pm}, X_{i_{2}, j_{2}, r_{2}+1}^{ \pm}\right] \text {, }} \\
& {\left[X_{i_{1}, j_{1}, r_{1}}^{+}, X_{i_{2}, j_{2}, r_{2}}^{-}\right]=\delta_{i_{1}=i_{2}} \delta_{j_{1}=j_{2}}\left(H_{i_{1}, j_{1}, r_{1}+r_{2}}+r_{1} \delta_{r_{1}+r_{2}=0} \mathrm{C}\right),} \\
& \operatorname{ad}\left(X_{i_{1}, j_{1}, r_{1}}^{ \pm}\right)^{1-c_{f\left(i_{1}, j_{1}\right), f\left(i_{2}, j_{2}\right)}}\left(X_{i_{2}, j_{2}, r_{2}}^{ \pm}\right)=0 .
\end{aligned}
$$

Remark 4.5. The elements with $r=0$ generate a copy of $\widehat{\mathfrak{s l}}_{n}\left(\mathbb{C}\left[u^{ \pm 1}\right] \rtimes \Gamma\right)$, and this proposition gives a set of relations describing this algebra, which is the one in terms of Chevalley-Kac generators of $\widehat{\mathfrak{s l}}_{n d}\left(\mathbb{C}\left[t^{ \pm 1}\right]\right)$ - see Proposition 3.8. The elements with $i \neq 0$ generate a central extension of $\mathfrak{s l}_{n}\left(\mathbb{C}\left[w^{ \pm 1}\right]\right)^{\oplus d}$, and this proposition gives a presentation of $\mathfrak{s l}_{n}\left(\mathbb{C}\left[w^{ \pm 1}\right]\right)$, which is the one obtained by considering a Cartan matrix of finite type $A_{n-1}$ in [Moody et al. 1990, Proposition 3.5].

An isomorphism $\tau: \mathfrak{t} \stackrel{\sim}{\longrightarrow} \widehat{\mathfrak{s l}}_{n}(A)$ is given explicitly on the generators by the following formulas

$$
\begin{aligned}
\left(X_{i, j, r}^{+}, X_{i, j, r}^{-}\right) & \left\{\begin{array}{lr}
\left(E_{i, i+1} w^{r} \mathbf{e}_{j}, E_{i+1, i} w^{r} \mathbf{e}_{j}\right) & \text { if } i \neq 0, \\
\left(E_{n 1} u^{-1} w^{r} \mathbf{e}_{j}, E_{1 n} u w^{r} \mathbf{e}_{j-1}\right) & \text { if } i=0, j \neq 0, \\
\left(E_{n 1} u^{2 d-1} w^{r} \mathbf{e}_{d-1}, E_{1 n} \otimes u^{-(2 d-1)} w^{r} \mathbf{e}_{0}\right) & \text { if } i=j=0,
\end{array}\right. \\
H_{i, j, r} & \mapsto \begin{cases}\left(E_{i i}-E_{i+1, i+1}\right) w^{r} \mathbf{e}_{j} & \text { if } i \neq 0, \\
E_{n n} w^{r} \mathbf{e}_{j-1}-E_{11} w^{r} \mathbf{e}_{j} & \text { if } i=0, j \neq 0, \\
E_{n n} w^{r} \mathbf{e}_{0}-E_{11} w^{r} \mathbf{e}_{d-1}-d w^{r} u^{-1} d u & \text { if } i=j=0,\end{cases} \\
\mathrm{c} & \mapsto w^{-1} d w=u^{-1} d u+v^{-1} d v .
\end{aligned}
$$

It is possible to obtain similar presentations for $\widehat{\mathfrak{s l}}_{n}(B)$ and $\widehat{\mathfrak{s l}}_{n}(C)$, which are Lie subalgebras of $\widehat{\mathfrak{s l}}_{n}(A)$.

4B. Derivations in the toroidal case. The Kac-Moody Lie algebras of affine type associated to the semisimple Lie algebra $\mathfrak{g}$ are obtained by adding a derivation to the universal central extension of $\mathfrak{g} \otimes_{\mathbb{C}} \mathbb{C}\left[t^{ \pm 1}\right]$. People who work on extended affine Lie algebras know to extend $\widehat{\mathfrak{s l}}_{n}\left(\mathbb{C}\left[s^{ \pm 1}, t^{ \pm 1}\right]\right)$ by adding derivations. Following [Allison et al. 1997], we interpret this in the context of $\mathfrak{s l}_{n}(A), \mathfrak{s l}_{m}(B), \mathfrak{s l}_{n}(C)$.

To $\widehat{\mathfrak{s l}}_{n}(A)$, we add derivations $\mathrm{d}_{u}$ and $\mathrm{d}_{w}^{v}$ that satisfy the commutation relations $\left[\mathrm{d}_{u}, m \otimes u^{k} w^{l} \gamma\right]=0 \quad$ if $k \neq 0 \bmod d$, $\left[\mathrm{d}_{u}, m \otimes u^{d k} v^{j} \gamma\right]=k m \otimes u^{d k} w^{j} \gamma$, $\left[\mathrm{d}_{w}^{v}, m \otimes u^{k} w^{l} \gamma\right]=l m \otimes u^{k} w^{l} \gamma$. 
We observe that

$$
\left[\mathrm{d}_{w}^{v}, m \otimes u^{k} v^{l} \gamma\right]=l m \otimes u^{k} v^{l} \gamma \quad \text { and } \quad\left[\mathrm{d}_{u}, m \otimes u^{k} v^{l} \gamma\right]=\delta_{k \equiv l} \frac{k-l}{d} m \otimes u^{k} v^{l} \gamma .
$$

We could define similarly $\mathrm{d}_{v}$ and $\mathrm{d}_{w}^{u}$. We then have the relation $\mathrm{d}_{u}=-\mathrm{d}_{v}$ and $d \cdot \mathrm{d}_{u}=\mathrm{d}_{w}^{u}-\mathrm{d}_{w}^{v}$. Dropping the index $w$, we can add two derivations $\mathrm{d}^{u}$ and $\mathrm{d}^{v}$ to $\widehat{\mathfrak{s l}}_{n}(A)$.

Definition 4.6. The cyclic double affine Lie algebra $\overline{\mathfrak{s l}}_{n}(A)$ is defined by adding the derivations $\mathrm{d}^{u}$ and $\mathrm{d}^{v}$ to $\widehat{\mathfrak{s l}}_{n}(A)$. We define similarly $\overline{\mathfrak{s l}}_{n}(B)$ and $\overline{\mathfrak{s l}}_{n}(C)$.

4C. Triangular decompositions. We have the triangular decompositions

$$
\mathfrak{s l}_{n}(A) \cong\left(\mathfrak{n}^{-} A\right) \oplus(\mathfrak{h} A \oplus I[A, A]) \oplus\left(\mathfrak{n}^{+} A\right),
$$

and $\mathfrak{s l}_{n}(A)$ is also isomorphic to

$$
\begin{aligned}
& \mathfrak{s l}_{n} u^{-1} \mathbb{C}\left[u^{-1}, v^{ \pm 1}\right] \rtimes \Gamma \oplus\left(\bigoplus_{\substack{i=1 \\
j \leq-1}}^{d-1} I\left(\mathbb{C}\left[v^{ \pm 1}\right] u^{j} \xi^{i}\right) \oplus \bigoplus_{\substack{s \leq-1 \\
r \neq s}} I\left(\mathbb{C} u^{s} v^{r}\right)\right) \\
& \oplus \mathfrak{n}^{-} \mathbb{C}\left[v^{ \pm 1}\right] \rtimes \Gamma \oplus\left(\mathfrak{h} \mathbb{C}\left[v^{ \pm 1}\right] \rtimes \Gamma \oplus\left(\bigoplus_{1 \leq i \leq d-1} I\left(\mathbb{C}\left[v^{ \pm 1}\right] \xi^{i}\right) \oplus \bigoplus_{r \neq 0} I\left(\mathbb{C} v^{r}\right)\right)\right) \\
& \oplus \mathfrak{s l}_{n} u \mathbb{C}\left[u, v^{ \pm 1}\right] \rtimes \Gamma \oplus\left(\bigoplus_{\substack{i=1 \\
j \geq 1}} I\left(\mathbb{C}\left[v^{ \pm 1}\right] u^{j} \xi^{i}\right) \oplus \bigoplus_{\substack{s \geq 1 \\
r \neq s}} I\left(\mathbb{C} u^{s} v^{r}\right)\right) \oplus \mathfrak{n}^{+} \mathbb{C}\left[v^{ \pm 1}\right] \rtimes \Gamma .
\end{aligned}
$$

and then by, setting $w=u v$, we see that $\mathfrak{s l}_{n}(A)$ is also isomorphic to

$$
\mathfrak{s l}_{n} u^{-1} \mathbb{C}\left[u^{-1}, w^{ \pm 1}\right] \rtimes \Gamma \oplus\left(\bigoplus_{\substack{i=1 \\ j \leq-1, r \in \mathbb{Z}}}^{d-1} I\left(\mathbb{C} w^{r} u^{j} \xi^{i}\right) \oplus \bigoplus_{\substack{s \leq-1 \\ s \neq \equiv, r \in \mathbb{Z}}} I\left(\mathbb{C} w^{r} u^{s}\right)\right)
$$

$\oplus \mathfrak{n}^{-} \mathbb{C}\left[w^{ \pm 1}\right] \rtimes \Gamma \oplus\left(\mathfrak{h} \mathbb{C}\left[w^{ \pm 1}\right] \rtimes \Gamma \oplus\left(\bigoplus_{1 \leq i \leq d-1} I\left(\mathbb{C}\left[w^{ \pm 1}\right] \xi^{i}\right)\right)\right) \oplus \mathfrak{s l}_{n} u \mathbb{C}\left[u, w^{ \pm 1}\right] \rtimes \Gamma$

$$
\oplus\left(\bigoplus_{\substack{i=1 \\ j \geq 1, r \in \mathbb{Z}}}^{d-1} I\left(\mathbb{C} w^{r} u^{j} \xi^{i}\right) \oplus \bigoplus_{\substack{s \geq 1 \\ s \neq 0, r \in \mathbb{Z}}} I\left(\mathbb{C} w^{r} u^{s}\right)\right) \oplus \mathfrak{n}^{+} \mathbb{C}\left[w^{ \pm 1}\right] \rtimes \Gamma .
$$

In the last two decompositions, one can exchange $u$ and $v$ and get other decompositions.

The universal central extensions $\widehat{\mathfrak{s l}}_{n}(A), \widehat{\mathfrak{s l}}_{n}(B)$ and $\widehat{\mathfrak{s l}}_{n}(C)$ also have three triangular decompositions They are obtained by adding the center to the middle part.

It is worth looking quickly at $\mathfrak{h} A \oplus I[A, A]$. We know that $A \cong M_{d}\left(\mathbb{C}\left[s^{ \pm 1}, t^{ \pm 1}\right]\right)$, so, if $d>1$, then $[A, A]=A$ and $\mathfrak{h} A \oplus I[A, A] \cong \mathfrak{g l}_{d}\left(\mathbb{C}\left[s^{ \pm 1}, t^{ \pm 1}\right]\right)^{\oplus n}$. 


\section{Representations of cyclic double affine Lie algebras}

In this section, we begin to study the representation theory of the algebras defined in the previous sections.

\section{A. Integrable and highest weight modules for cyclic double affine Lie algebras.}

We have just presented three triangular decompositions of $\widehat{\mathfrak{s l}}_{n}(A)$. The first one is analogous to the one used for Weyl modules in [Feigin and Loktev 2004], but in our situation the middle Lie algebra is not commutative if $\Gamma \neq\{\mathrm{id}\}$. (Note that an analogue of the first triangular decomposition is not known for quantum toroidal algebras.) We will return to Weyl modules in Section 5B below.

The second triangular decomposition corresponds to the first triangular decomposition of the cyclic affine Lie algebras for the parameter $v$, and to the second of the cyclic affine Lie algebra for the parameter $u$. Thus it is analogous to the triangular decomposition used in [Miki 2000; Nakajima 2001; Hernandez 2005] to construct $l$-highest weight representations of quantum toroidal algebras. Again, in our situation, the middle Lie algebra is not commutative if $\Gamma \neq\{\mathrm{id}\}$.

However, the middle term $\mathfrak{H}$ of the last triangular decomposition is a commutative Lie algebra. Actually, this last case is obtained by considering the first triangular decomposition of the cyclic affine Lie algebras for the parameter $w$ and the second triangular decomposition of the cyclic affine Lie algebra for the parameter $u$. Under the isomorphism between $\widehat{\mathfrak{s l}}_{n}(A)$ and the toroidal Lie algebra $\widehat{\mathfrak{s l}}_{n d}\left(\mathbb{C}\left[u^{ \pm 1}, w^{ \pm 1}\right]\right)$ given in Proposition 4.1, it corresponds to the standard decomposition of $\widehat{\mathfrak{s l}}_{n d}\left(\mathbb{C}\left[u^{ \pm 1}, w^{ \pm 1}\right]\right)$ as used in [Chari and Le 2003] for a certain central extension of $\mathfrak{s l}_{n d}\left(\mathbb{C}\left[u^{ \pm 1}, w^{ \pm 1}\right]\right)$. (It is analogous to the decomposition considered in the quantum case [Miki 2000; Nakajima 2001; Hernandez 2005]). In particular, the notions of integrable and highest weight modules for this decomposition have been studied in [Chari and Le 2003; Rao 2004; Yoon 2002]; we simply reformulate their results for the benefit of the reader in the next subsection. Integrable highest weight representations are classified by tuples of $n d-1$ polynomials. In opposition to the quantum case, evaluation morphisms are available and provide a direct way to construct integrable representations.

The standard highest weight structure on $\widehat{\mathfrak{s l}}_{n}(A)$ and $\widehat{\mathfrak{s l}}_{n}(B)$. In this subsection, we include previously known results about the standard highest weight structure on $\widehat{\mathfrak{s l}}_{n}(A)$ and on $\widehat{\mathfrak{s l}}_{n}(B)$. Actually, the results below have been proved only for $\widehat{\mathfrak{s l}}_{n}(A)$, but the proofs are similar for $\widehat{\mathfrak{s l}}_{n}(B)$. Let $\mathfrak{g}^{ \pm}$be the positive and negative parts of the triangular decomposition (3), and let $\mathfrak{H}$ be the middle part.

Instead of highest weight vectors, we have to consider the notion of pseudohighest weight vectors. Suppose that

$$
\Lambda=\left(\lambda_{i, j, r}\right)_{0 \leq i \leq n-1, r \in \mathbb{Z}}^{0 \leq j \leq d-1}, \quad \text { where } \lambda_{i, j, r} \in \mathbb{C} .
$$


We define the Verma module $M(\Lambda)$ to be the $\widehat{\mathfrak{s l}}_{n}(A)$-module induced from the $\mathfrak{H} \oplus \mathfrak{g}^{+}$representation generated by the vector $v_{\Lambda}$ on which $\mathfrak{g}^{+}$and $\mathrm{c}$ act by zero and $H_{i, j, r}$ acts by multiplication by $\lambda_{i, j, r}$ (in the notation of Proposition 4.4). We have a grading on the Verma module and so it has a unique simple quotient $L(\lambda)$. For $\mu \in \mathfrak{H}^{*}$, we define the notion of weight space $V_{\mu}$ of a representation $V$ as usual.

Definition 5.1. A module $M$ over $\widehat{\mathfrak{s l}}_{n}(A)$ is integrable if $M=\bigoplus_{\mu \in \mathfrak{H}^{*}} M_{\mu}$ and if the vectors $E_{i j} u^{s} v^{r} \gamma$ act locally nilpotently if $1 \leq i \neq j \leq n, r, s \in \mathbb{Z}$ and $\gamma \in \Gamma$.

Note that, in the quantum case, a stronger notion of integrability is used instead of local nilpotency of the operators [Chari and Le 2003; Rao 2004].

Proposition 5.2 [Chari and Le 2003]. The irreducible module $L(\Lambda)$ is integrable if and only if, for any $0 \leq i \leq n-1$ and $0 \leq j \leq d-1$, we have $\lambda_{i, j, 0} \in \mathbb{Z}_{\geq 0}$ and there exist monic polynomials $P_{i, j}(z)$ of degree $\lambda_{i, j, 0}$ such that

$$
\sum_{r \geq 1} \lambda_{i, j, r} z^{r-1}=-\frac{P_{i, j}^{\prime}(z)}{P_{i, j}(z)} \quad \text { and } \quad \sum_{r \geq 1} \lambda_{i, j,-r} z^{r-1}=-\lambda_{i, j, 0} z^{-1}+z^{-2} \frac{P_{i, j}^{\prime}\left(z^{-1}\right)}{P_{i, j}\left(z^{-1}\right)}
$$

as formal power series.

The proof of the necessary condition in this proposition reduces to the case of the loop Lie algebra of $\mathfrak{s l}_{2}$, which is why it extends automatically from the affine to the double affine setup. The sufficiency is proved as in [Chari and Le 2003] using tensor products of evaluation modules (the formulas for the power series are a bit different from those in [Chari and Le 2003, Proposition 3.1] as we use difference variables; see also [Miki 2004]). In the quantum context, the polynomials $P_{i, j}(z)$ are called Drinfeld polynomials. A similar criterion for integrability exists for quantum toroidal algebras; this is explained in [Hernandez 2005] after proving that certain subalgebras of a quantum toroidal algebras are isomorphic to the quantized enveloping algebra of $\mathfrak{s l}_{2}\left(\mathbb{C}\left[t^{ \pm 1}\right]\right)$. Affine Yangians for $\mathfrak{s l}_{n}$ are built from copies of $Y\left(\mathfrak{s l}_{2}\right)$, the Yangian for $\mathfrak{s l}_{2}$ : this follows from the PBW property of affine Yangians proved in [Guay 2007]; hence a similar integrability condition holds for them also.

The standard highest weight structure on $\widehat{\mathfrak{s l}}_{n}(C)$. The case that interests us more and presents some novelty is $\widehat{\mathfrak{s l}}_{n}(C)$, because the Lie algebra $\mathfrak{s l}_{n}(C)$ is not isomorphic to $\mathfrak{s l}_{n d}(\mathbb{C}[s, t])$. The three triangular decompositions of $\widehat{\mathfrak{s l}}_{n}(A)$ given at the end of the Section 4 yield such decompositions for $\widehat{\mathfrak{s l}}_{n}(C)$, and we consider the one coming from (3). More precisely, $\mathfrak{s l}_{n}(C)$ can be decomposed into the direct 
sum of three subalgebras, as

$$
\text { (4) } \begin{aligned}
&\left(\mathfrak{s l}_{n} v \mathbb{C}[v, w] \rtimes \Gamma \oplus\left(\bigoplus_{\substack{j \geq 1 \\
1 \leq i \leq d-1}} I\left(\mathbb{C}[w] v^{j} \xi^{i}\right) \oplus \bigoplus_{\substack{r \geq 1 \\
r \neq 0}} I\left(\mathbb{C}[w] v^{r}\right)\right) \oplus \mathfrak{n}^{-} \mathbb{C}[w] \rtimes \Gamma\right) \\
& \oplus\left(\mathfrak{h} \mathbb{C}[w] \rtimes \Gamma \oplus\left(\bigoplus_{1 \leq i \leq d-1} I\left(w \mathbb{C}[w] \xi^{i}\right)\right)\right) \\
& \oplus\left(\mathfrak{s l}_{n} u \mathbb{C}[u, w] \rtimes \Gamma \oplus\left(\underset{\substack{j \geq 1 \\
1 \leq i \leq d-1}}{\bigoplus} I\left(\mathbb{C}[w] u^{j} \xi^{i}\right) \oplus \underset{\substack{r \geq 1 \\
r \neq 0}}{\bigoplus} I\left(\mathbb{C}[w] u^{r}\right)\right) \oplus \mathfrak{n}^{+} \mathbb{C}[w] \rtimes \Gamma\right) .
\end{aligned}
$$

We have an embedding $\widehat{\mathfrak{s l}}_{n}(C) \hookrightarrow \widehat{\mathfrak{s l}}_{n d}\left(\mathbb{C}\left[u^{ \pm 1}, v\right]\right)$, but in order to classify integrable, highest weight representations of $\widehat{\mathfrak{s l}}_{n}(C)$, we will instead use the following presentation.

Proposition 5.3. The Lie algebra $\widehat{\mathfrak{s l}}_{n}(C)$ is isomorphic to the Lie algebra $\mathfrak{k}$ that is generated by the elements $X_{i, j, r}^{ \pm}, H_{i, j, r}, X_{0, j, r}^{+}, X_{0, j, r+1}^{-}, H_{0, j, r+1}$ for $1 \leq i \leq n-1$, $0 \leq j \leq d-1$ and $r \in \mathbb{Z}_{\geq 0}$, and that satisfies the relations

$$
\begin{aligned}
{\left[H_{i_{1}, j_{1}, r_{1}}, H_{i_{2}, j_{2}, r_{2}}\right] } & =0, \\
{\left[H_{i_{1}, j_{1}, 0}, X_{i_{2}, j_{2}, r_{2}}^{ \pm}\right] } & = \pm c_{f\left(i_{1}, j_{1}\right), f\left(i_{2}, j_{2}\right)} X_{i_{2}, j_{2}, r_{2}}^{ \pm}, \\
{\left[H_{i_{1}, j_{1}, r_{1}+1}, X_{i_{2}, j_{2}, r_{2}}^{ \pm}\right] } & =\left[H_{i_{1}, j_{1}, r_{1}}, X_{i_{2}, j_{2}, r_{2}+1}^{ \pm}\right], \\
{\left[X_{i_{1}, j_{1}, r_{1}+1}^{ \pm}, X_{i_{2}, j_{2}, r_{2}}^{ \pm}\right] } & =\left[X_{i_{1}, j_{1}, r_{1}}^{ \pm}, X_{i_{2}, j_{2}, r_{2}+1}^{ \pm}\right], \\
{\left[X_{i_{1}, j_{1}, r_{1}}^{+}, X_{i_{2}, j_{2}, r_{2}}^{-}\right] } & =\delta_{i_{1}=i_{2}} \delta_{j_{1}=j_{2}} H_{i_{1}, j_{1}, r_{1}+r_{2}}, \\
\operatorname{ad}\left(X_{i_{1}, j_{1}, r_{1}}^{ \pm}\right)^{1-c_{f\left(i_{1}, j_{1}\right), f\left(i_{2}, j_{2}\right)}}\left(X_{i_{2}, j_{2}, r_{2}}^{ \pm}\right) & =0 .
\end{aligned}
$$

The proof of Proposition 4.4 in [Moody et al. 1990] works also for $\widehat{\mathfrak{s l}}_{n}(B)$, and it is possible to deduce from it Proposition 5.3. See [Guay 2009b] for more details.

The elements with $i \neq 0$ generate a Lie subalgebra isomorphic to $\mathfrak{s l}_{n}(\mathbb{C}[w] \rtimes \Gamma)$, the elements $X_{i, j, r}^{+}$for $0 \leq i \leq n-1,0 \leq j \leq d-1$ and $r \in \mathbb{Z}_{\geq 0}$ generate the positive part $\widehat{\mathfrak{s l}}_{n}(C)^{+}$of the decomposition (4), and, finally, the elements $X_{i, j, r}^{-}$and $X_{0, j, r+1}^{-}$ for $0 \leq i \leq n-1,0 \leq j \leq d-1$ and $r \in \mathbb{Z}_{\geq 0}$ generate the decomposition's negative part $\widehat{\mathfrak{s l}}_{n}(C)^{-}$. Note that $\widehat{\mathfrak{s l}}_{n}(C)^{+} \cong \widehat{\mathfrak{s l}}_{n}(C)^{-}$via $X_{i, j, r}^{+} \mapsto X_{i, j, r}^{-}$for $1 \leq i \leq n-1$ and $X_{0, j, r}^{+} \mapsto X_{0, j, r+1}^{-}$. The elements with $r=0$ generate a copy of $\mathfrak{s l}_{n}(\mathbb{C}[u] \rtimes \Gamma)$, whereas the elements $X_{i, j, 0}^{ \pm}$and $X_{0, j, 1}^{-}$with $1 \leq i \leq n-1$ and $0 \leq j \leq d-1$ generate a Lie subalgebra isomorphic to $\mathfrak{s l}_{n}(\mathbb{C}[v] \rtimes \Gamma)$. For a fixed $0 \leq j \leq d-1$, the elements $X_{0, j, r}^{+}, X_{0, j, r+1}^{-}, H_{0, j, r+1}$ for all $r \in \mathbb{Z}_{\geq 0}$ generate a subalgebra of $\mathfrak{s l}_{2}(\mathbb{C}[w])$ which, as a vector space, is $\mathfrak{n}_{2}^{-} w \mathbb{C}[w] \oplus \mathfrak{h}_{2} w \mathbb{C}[w] \oplus \mathfrak{n}_{2}^{+} \mathbb{C}[w]$, where the subscript 2 labels the corresponding subalgebra of $\mathfrak{s l}_{2}$. We denote this subalgebra of $\mathfrak{s l}_{2}(\mathbb{C}[w])$ by $\mathfrak{s}_{2}(\mathbb{C}[w])$. 
Integrability of representations of $\widehat{\mathfrak{s l}}_{n}(C)$ has the same meaning as it did in Definition 5.1. As for $\widehat{\mathfrak{s l}}_{n}(A)$ and $\widehat{\mathfrak{s l}}_{n}(B)$, we have Verma modules $M(\Lambda)$ and their irreducible quotients $L(\Lambda)$ for each pseudoweight $\Lambda=\left(\lambda_{i, j, r} \in \mathbb{C}\right)$ with $0 \leq i \leq n-1, r \in \mathbb{Z}$ and $0 \leq j \leq d-1$ but $r \geq 1$ if $i=0$; the highest weight cyclic generator is again denoted $v_{\Lambda}$.

Proposition 5.4. The irreducible module $L(\Lambda)$ is integrable if and only if $\lambda_{i, j, 0}$ belongs to $\mathbb{Z}_{\geq 0}$ for any $1 \leq i \leq n-1$ and $0 \leq j \leq d-1$, and there exist monic polynomials $P_{i, j}(z)$ for the same range of $i$ and $j$ such that $\sum_{r \geq 1} \lambda_{i, j, r} z^{-r-1}$ is equal to $-\operatorname{deg}\left(P_{i, j}\right)+P_{i, j}^{\prime}(z) / P_{i, j}(z)$ as formal power series and $P_{i, j}(z)$ is of degree $\lambda_{i, j, 0}$ if $1 \leq i \leq n-1$ and $0 \leq j \leq d-1$.

Let us say a few words about the proof. The main difference with the cases $\widehat{\mathfrak{s l}}_{n}(A)$ and $\widehat{\mathfrak{s l}}_{n}(B)$ is that we do not have the elements $X_{0, j, 0}^{-}$for $0 \leq j \leq d-1$. However, it is still possible to apply argument of Chari [1986] in the affine $\mathfrak{s l}_{2}$ case, modulo some small differences. For instance, [Chari 1986, Proposition 1.1] is fundamental for the rest of that article, but it cannot be applied in our case when $i=0$. What we need instead is an expression for $\left(X_{0, j, 0}\right)^{r}\left(X_{0, j, 1}^{-}\right)^{r}$. But Chari's proposition is a consequence of [Garland 1978, Lemma 7.5]. To obtain an expression for $\left(X_{0, j, 0}\right)^{r}\left(X_{0, j, 1}^{-}\right)^{r}$, we just have to apply the automorphism of $\check{\mathfrak{s}}_{2}(\mathbb{C}[w])$ given by

$$
E_{21} w^{r+1} \mapsto E_{12} w^{r}, \quad E_{12} w^{r} \mapsto E_{21} t^{r+1}, \quad\left(E_{11}-E_{22}\right) t^{r+1} \mapsto\left(E_{22}-E_{11}\right) t^{r+1}
$$

for $r \in \mathbb{Z}_{\geq 0}$. We lose the condition that the degree of $P_{0, j}(z)$ is $\lambda_{0, j}$ (here the degree of $P_{0, j}(z)$ is the smallest integer $r$ such that $\left.\left(X_{0, j, 1}^{-}\right)^{r+1} v_{\Lambda}=0\right)$ because $\widehat{\mathfrak{s l}}_{n}(C)$ does not contain the $\mathfrak{s l}_{2}$-copies generated by $X_{0, j, 0}^{ \pm}$and $H_{0, j, 0}$. The proof of the sufficiency of the condition in the proposition consists in the construction of an integrable quotient of the Verma module $M(\Lambda)$ using tensor products of evaluation modules, as in [Chari and Le 2003]. Note that also in the case of $\widehat{\mathfrak{s l}}_{n}(C)$, the degree of $P_{0, j}(z)$ is the smallest integer $r$ such that $\left(X_{0, j, 1}^{-}\right)^{r+1}$ acts by zero on the cyclic highest weight vector.

5B. Weyl modules for $\mathfrak{s l}_{n}(\mathbb{C}[u, v] \rtimes \Gamma)$. For a Lie algebra $\mathfrak{g}$ and a commutative $\mathbb{C}$ algebra $\mathscr{A}$, we may denote $\mathfrak{g} \otimes_{\mathbb{C}} \mathscr{A}$ by $\mathfrak{g}(\mathscr{A})$ or $\mathfrak{g} \mathscr{A}$. If $\mathfrak{g}=\mathfrak{n}^{+} \oplus \mathfrak{H} \oplus \mathfrak{n}^{-}$, the Weyl modules [Feigin and Loktev 2004] are certain representations of $\mathfrak{g}(\mathscr{A})$ generated by a weight vector $v$ satisfying $\left(\mathfrak{n}^{+}(\mathscr{A})\right) \cdot v=0$. (In this subsection, we consider only the local Weyl modules, not the global ones.) The motivation to study Weyl modules is that they should be simpler to understand than the finite-dimensional irreducible modules. This is what happens in the quantum affine setup where the Weyl modules for the affine Lie algebras are closely related to finite-dimensional irreducible modules of the corresponding affine quantum group when $q \mapsto 1$; see [Chari and Pressley 2001; Chari and Loktev 2006]. The definition of Weyl modules depends 
on the choice of a triangular decomposition, but only the first of our triangular decompositions for cyclic double affine Lie algebras seems appropriate. It should be noted that we cannot use Proposition 4.1 to deduce results about Weyl modules for $\mathfrak{s l}_{n}(A)$ in our context because, when $\Gamma$ is nontrivial, the isomorphism in that proposition does not map the triangular decomposition (1) of $\mathfrak{s l}_{n}(A)$ to the decomposition $\mathfrak{s l}_{n d}\left(\mathbb{C}\left[s^{ \pm 1}, t^{ \pm 1}\right]\right)=\mathfrak{n}_{n d}^{-} \mathbb{C}\left[s^{ \pm 1}, t^{ \pm 1}\right] \oplus \mathfrak{h}_{n d} \mathbb{C}\left[s^{ \pm 1}, t^{ \pm 1}\right] \oplus \mathfrak{n}_{n d}^{+} \mathbb{C}\left[s^{ \pm 1}, t^{ \pm 1}\right]$ considered in [Feigin and Loktev 2004].

In this subsection, we need a definition of integrability stronger than the one used in Definition 5.1, namely, the one used in [Feigin and Loktev 2004].

Definition 5.5. A module $M$ over a Lie algebra of the type $\mathfrak{g} \otimes_{\mathbb{C}} \mathscr{A}$ is said to be integrable if $M_{\mu}$ is nonzero for only finitely many $\mu \in P$.

When $\mathscr{A}$ is the coordinate ring of an affine algebraic variety $X$, Weyl modules are associated to multisets of points of $X$. In the simplest case of a (closed) point, we have an augmentation $\mathscr{A} \rightarrow \mathbb{C}$. However, when it comes to the triangular decomposition (1), the middle term is isomorphic to $\mathfrak{d} \otimes_{\mathbb{C}} M_{d}\left(\mathbb{C}\left[s^{ \pm 1}, t^{ \pm 1}\right]\right)$, where $\mathfrak{d}$ is the abelian Lie algebra of the diagonal matrices in $\mathfrak{g l}_{n}$. When $d=1$, we are exactly in the same situation as in [Feigin and Loktev 2004] (with $X$ the twodimensional torus $\mathbb{C}^{\times} \times \mathbb{C}^{\times}$), but when $d>1$, the Lie algebra is noncommutative. One new possibility is to consider maximal two-sided ideals in $A \rtimes \Gamma$ or, equivalently, augmentation maps. We are thus led to the following definition, which we can formulate in a more general setting.

Definition 5.6. Let $\mathscr{A}$ be a commutative, finitely generated algebra with a unit, and let $G$ be a finite group acting on $\mathscr{A}$ by algebra automorphisms. Consider an augmentation $\epsilon$ of $\mathscr{A} \rtimes G$, that is, an algebra homomorphism $\mathscr{A} \rtimes G \rightarrow \mathbb{C}$, and let $\lambda \in \mathfrak{h}^{*}$ be a dominant integral weight. We define the Weyl module $W_{\mathscr{A} \rtimes G}^{\epsilon}$ to be the maximal integrable cyclic $\mathfrak{s l}_{n}(\mathscr{A} \rtimes G)$-module generated by a vector $v_{\lambda}$ such that, for $a \in \mathscr{A} \rtimes G$,

$$
(h a)\left(v_{\lambda}\right)=\lambda(h) \epsilon(a) v_{\lambda} \quad \text { and } \quad \mathfrak{n}^{+}(\mathscr{A} \rtimes G)\left(v_{\lambda}\right)=0 .
$$

The existence of such a maximal module can be proved as in [Feigin and Loktev 2004] using the notion of global Weyl module. This definition agrees with the one used in that paper in the case $G=\{\mathrm{id}\}$. We note that, by $\mathfrak{s l}_{2}$-theory, we have that $f_{i}^{\lambda\left(h_{i}\right)+1} v_{\lambda}=0$, where, as usual, we denote by $f_{i}, h_{i}, e_{i}$ for $1 \leq i \leq n-1$ the standard Chevalley generators of $\mathfrak{s l}_{n}$.

It turns out that Weyl modules for the smash product $\mathscr{A} \rtimes G$ are related to Weyl modules for a much smaller ring. We have a decomposition of $G$-modules $\mathscr{A}=\mathscr{A}^{G} \oplus \mathscr{A}^{\prime}$, where $\mathscr{A}^{\prime}$ is the subrepresentation without invariants. Let us denote by $\bar{A}$ the quotient of $\mathscr{A}$ by the two-sided ideal generated by $\mathscr{A}^{\prime}$. Note that it may be 
much smaller than $\mathscr{A}^{G}$ - it can even reduce to $\mathbb{C}$, for instance, when $G$ is $\mathbb{Z} / d \mathbb{Z}$ and $\mathscr{A}=\mathbb{C}[u]$ or even $\mathscr{A}=\mathbb{C}[u, v]$.

Consider an augmentation $\epsilon$ of $\mathscr{A} \rtimes G$. Note that $\mathscr{A}^{\prime} \subset[\mathscr{A} \rtimes G, \mathscr{A} \rtimes G]$, so $\epsilon\left(\mathscr{A}^{\prime}\right)=0$ and $\epsilon$ descends to an augmentation $\bar{\epsilon}$ of $\bar{A}$.

Theorem 5.7. Let $\lambda \in \mathfrak{h}^{*}$ be a dominant integral weight. We have an isomorphism of modules over $\mathfrak{s l}_{n}(\mathscr{A} \rtimes G)$ given by

$$
W_{\mathscr{A} \rtimes \mathbb{C}[G]}^{\epsilon}(\lambda) \cong W_{\mathscr{A}}^{\bar{\epsilon}}(\lambda) .
$$

We have a surjective map $\mathscr{A} \rtimes G \rightarrow \bar{A}$; hence $\mathfrak{s l}_{n}(\mathscr{A} \rtimes G)$ acts on $W_{\mathscr{A}}^{\bar{\epsilon}}(\lambda)$ and this yields a surjective map $W_{\mathscr{A} \rtimes G}^{\epsilon}(\lambda) \rightarrow W_{\bar{A}}^{\bar{\epsilon}}(\lambda)$ of modules over $\mathfrak{s l}_{n}(\mathscr{A} \rtimes G)$. The ring $\bar{A}$ is the quotient of $\mathscr{A} \rtimes G$ by the ideal $J_{\epsilon}$ generated by $\mathscr{A}^{\prime}$ and elements $\gamma-\epsilon(\gamma)$ in $\mathbb{C}[G]$ for $\gamma \in G$. We need only show that $\mathfrak{s l}_{n} \otimes J_{\epsilon}$ acts on $W_{\mathscr{A} \rtimes G}^{\epsilon}(\lambda)$ by zero.

Actually, since $\mathfrak{s l}_{n} \otimes J_{\epsilon}$ is an ideal, it is enough to show that it acts by zero on the highest weight vector $v_{\lambda}$. As $\epsilon\left(J_{\epsilon}\right)=0$, this is true for $\mathfrak{b} \otimes J_{\epsilon}$, so it remains to prove our claim for $E_{i j} \otimes \epsilon$ with $i>j$. Now the question is reduced to $\mathfrak{s l}_{2}$-case, so, to simplify the notation, let us set $f=E_{i j}, e=E_{j i}$ and $h=E_{i i}-E_{j j}$.

Lemma 5.8. Let $P \in \mathscr{A} \rtimes \Gamma$. If $\epsilon(P)=0$, then $\left(f \otimes P^{\lambda(h)}\right) v_{\lambda}=0$.

Proof. We already know that $f^{\lambda(h)+1} v_{\lambda}=0$. Applying $e \otimes P$ a total of $j$ times and using the assumption $\epsilon(P)=0$ yields $\left(f^{\lambda(h)+1-j} \otimes P^{j}\right) v_{\lambda}=0$, so taking $j=\lambda(h)$ proves the lemma.

Lemma 5.9. If $(f \otimes P) v_{\lambda}=0$ and $Q \in \mathscr{A}$, then $(f \otimes(P Q+Q P)) v_{\lambda}=0$. Also, if $Q$ belongs to the commutator $\left[\mathfrak{s l}_{n}(\mathscr{A} \rtimes G), \mathfrak{s l}_{n}(\mathscr{A} \rtimes G)\right]$, then we have $(f \otimes P Q) v_{\lambda}=(f \otimes Q P) v_{\lambda}=0$.

Proof. Applying $f \otimes P$ to both sides of $(h \otimes Q) v_{\lambda}=\lambda(h) \epsilon(Q) v_{\lambda}$ yields the first equality. If now, say, $Q=Q_{1} Q_{2}-Q_{2} Q_{1}$, then $\left(E_{i i}+E_{j j}\right) \otimes Q=\left[h \otimes Q_{1}, h \otimes Q_{2}\right]$; starting from $(f \otimes Q) v_{\lambda}=0$ and applying $E_{i i} \otimes Q$ or $E_{j j} \otimes Q$ to both sides, we obtain the second equality. (Note here that $E_{i i} \otimes Q$ belongs to $\mathfrak{s l}_{2}(\mathscr{A} \rtimes G)$ since, by assumption, $Q \in\left[\mathfrak{s l}_{n}(\mathscr{A} \rtimes G), \mathfrak{s l}_{n}(\mathscr{A} \rtimes G)\right]$.)

Proof of Theorem 5.7. First let us show that

$$
(f \otimes(x-\epsilon(x))) v_{\lambda}=0 \quad \text { for } x \in \mathbb{C}[G] .
$$

Note that $\mathbb{C}[G]=\mathbb{C} c_{\epsilon} \oplus I_{\epsilon}$, where $c_{\epsilon}^{2}=c_{\epsilon}, \epsilon\left(c_{\epsilon}\right)=1$, and $I_{\epsilon}$ is the kernel of $\left.\epsilon\right|_{\mathbb{C}[G]}$. We have $I_{\epsilon}^{l+1}=I_{\epsilon}$, so Lemma 5.8 yields Equation (5) for $x \in I_{\epsilon}$. Also note that $c_{\epsilon}-1$ belongs to $I_{\epsilon}$, so we have (5) also for $x=c_{\epsilon}$ and, therefore, for any $x$.

Since $\mathscr{A}^{\prime} \in\left[\mathfrak{s l}_{n}(\mathscr{A} \rtimes G), \mathfrak{s l}_{n}(\mathscr{A} \rtimes G)\right]$ and $\mathscr{A}^{G}$ commutes with $\mathbb{C}[G]$, Lemma 5.9 implies that $(f \otimes \mathscr{A}(x-\epsilon(x))) v_{\lambda}=0$. 
It remains to show that $\left(f \otimes \mathscr{A}^{\prime}\right) v_{\lambda}=0$. By Lemma 5.9, for any $a \in \mathscr{A}^{\prime}$ and $\gamma \in G$, we have

$$
(f \otimes \gamma a) v_{\lambda}=(f \otimes a \gamma) v_{\lambda}=(f \otimes a \epsilon(\gamma)) v_{\lambda} .
$$

So

$$
\left(f \otimes\left(a-\gamma^{-1}(a)\right)\right) v_{\lambda}=\frac{1}{\epsilon(\gamma)}(f \otimes(\gamma a-a)) v_{\lambda}=0 .
$$

Finally, note that the elements $\left(a-\gamma^{-1}(a)\right)$ for $a \in \mathscr{A}^{\prime}$ and $\gamma \in G$ span $\mathscr{A}^{\prime}$.

5C. Weyl modules associated to rings of invariants. When $\mathscr{A}$ is a commutative, unital, finitely generated $\mathbb{C}$-algebra, Weyl modules for $\mathfrak{s l}_{n} \otimes_{\mathbb{C}} \mathscr{A}$ can be attached to multisets of points in $\operatorname{Spec}(\mathscr{A})$ or, more generally, to any ideal in $\mathscr{A}$. In this subsection, we first apply an approach due to Feigin and Loktev [2004] and Chari and Pressley [2001] to describe certain local Weyl modules for $\mathfrak{s l}_{n}(\mathbb{C}[u, v])$ and $\mathfrak{s l}_{n}\left(\mathbb{C}[u, v]^{\Gamma}\right)$. This approach realizes them as the Schur-Weyl duals of certain modules of coinvariants. A natural question to ask is, What is the dimension of these local Weyl modules? For loop algebras $\mathfrak{s l}_{n}\left(\mathbb{C}\left[u, u^{-1}\right]\right)$, this question was fully answered in [Chari and Loktev 2006]. For $\mathfrak{s l}_{n}(\mathbb{C}[u, v])$ and a multiple of the fundamental weight of the natural representation of $\mathfrak{s l}_{n}$ on $\mathbb{C}^{n}$, this problem was solved in [Feigin and Loktev 2004], but the answer relies on the difficult theorem of M. Haiman [2002] on the dimension of diagonal harmonics. To compute the dimension of the Weyl modules that we introduce below, we would need an extension of Haiman's theorem to certain rings of coinvariants attached to wreath products of the cyclic group $\mathbb{Z} / d \mathbb{Z}$, but this is still an open problem as far as we know. At least, we are able to provide a lower bound for the dimension of some local Weyl modules by using a theorem of R. Vale [2007], which generalizes an earlier result of I. Gordon [2003].

Definition 5.10. Let $U$ be a representation of $\mathfrak{s l}_{n} \otimes \mathscr{A}$ and let $\mu \in \mathfrak{h}^{*}$. Suppose that we have an augmentation map $\epsilon: \mathscr{A} \rightarrow \mathbb{C}$. A vector $v_{\mu} \in U$ is called a highest weight vector if $(g \otimes a) v_{\mu}=0$ when $g \in \mathfrak{n}^{+}, a \in \mathscr{A}$ and $(h \otimes a) v_{\mu}=\mu(h) \epsilon(a) v_{\mu}$ for all $h \in \mathfrak{h}$ and $a \in \mathscr{A}$.

Theorem 5.11 [Feigin and Loktev 2004]. Let $\mu \in \mathfrak{h}^{*}$ be a dominant integral weight. There exists a universal finite-dimensional module $W_{\epsilon}^{A A}(\mu)$ such that any finite-dimensional representation of $\mathfrak{s l}_{n} \otimes \mathscr{A}$ generated by a highest weight vector $v_{\mu}$ is a quotient of $W_{\epsilon}^{A}(\mu)$.

Recall a general result of [Feigin and Loktev 2004]; see also [Loktev 2008]. The symmetric group $S_{l}$ acts on $\mathscr{A}^{\otimes l}$, so we can form $\mathrm{DH}_{l}(\mathscr{A})=\mathscr{A}^{\otimes l} /\left(\operatorname{Sym}^{l}(\mathscr{A})_{\epsilon}\right)$, the quotient of $\mathscr{A}^{\otimes l}$ by the ideal generated by the tensors that are invariant under the action of $S_{l}$ and that are in the kernel of $\epsilon$ (extended as an augmentation $\operatorname{Sym}(\mathscr{A})$ ). When $\mathscr{A}=\mathbb{C}[u, v]$, this quotient is called the space of diagonal coinvariants. 
If $E$ is a representation of $S_{l}$, denote by $\mathrm{SW}_{l}^{n}(E)$ the representation of $\mathfrak{s l}_{n}$ given by $\left(\left(\mathbb{C}^{n}\right)^{\otimes l} \otimes E\right)^{S_{l}}$. This is the classical Schur-Weyl construction. Note that $\mathrm{DH}_{l}(\mathscr{A})$ is a representation of $S_{l}$. More generally, as is observed in [Loktev 2008], if $E$ is a representation of the smash product $\left(\mathscr{A}^{\otimes l}\right) \rtimes S_{l}$, then $\mathrm{SW}_{l}^{n}(E)$ is a representation of $\mathfrak{s l}_{n}(\mathscr{A})$.

Let $\omega_{1}$ be the fundamental weight of $\mathfrak{s l}_{n}$ that is the highest weight of its natural representation $\mathbb{C}^{n}$.

Theorem 5.12 [Feigin and Loktev 2004]. The Weyl module $W_{\epsilon}^{\mathscr{A}}\left(l \omega_{1}\right)$ of $\mathfrak{s l}_{n}(\mathscr{A})$ is isomorphic to $\mathrm{SW}_{l}^{n}\left(\mathrm{DH}_{l}(\mathscr{A})\right)$.

When $\mathscr{A}=\mathbb{C}[u, v]$, the dimension of the ring of diagonal harmonics $\mathrm{DH}_{l}(\mathscr{A})$ is a difficult result proved by M. Haiman [2002]. Until after Proposition 5.15, we will assume that $\Gamma$ is an arbitrary finite subgroup of $\mathrm{SL}_{2}(\mathbb{C})$. The preceding theorem can be applied to $\mathscr{A}=\mathbb{C}[u, v]^{G}$ and $\epsilon: \mathbb{C}[u, v]^{G} \rightarrow \mathbb{C}$, the homomorphism given by the maximal ideal $\mathbb{C}[u, v]_{+}^{G}$ corresponding to the singularity. (Here, $G$ is an arbitrary finite subgroup of $\mathrm{SL}_{2}(\mathbb{C})$.) It gives a nice description of the Weyl module for multiples of $\omega_{1}$, but, to compute its dimension, we would have to know more about the structure of $\mathrm{DH}_{l}\left(\mathbb{C}[u, v]^{G}\right)$ as a module for $S_{l}$. As far as we know, this is still an open problem when $G \neq\{1\}$. We are, however, able to obtain a partial result by considering the ring $\mathscr{A}=\mathbb{C}[u, v]$ but with highest weight conditions on $\mathfrak{h} \otimes \mathbb{C}[u, v]^{\Gamma}$, and from it we can deduce a lower bound when $\mathscr{A}=\mathbb{C}[u, v]^{\Gamma}$.

Denote by $H_{G, l}$ the quotient of $\mathbb{C}\left[u_{1}, \ldots, u_{l}, v_{1}, \ldots, v_{l}\right]$ by the ideal generated by $S_{l} \ltimes G^{\times l}$-invariants with zero at the origin. This is a module for $S_{l} \ltimes G^{\times l}$ and also for $\left(S_{l} \ltimes G^{\times l}\right) \ltimes \mathbb{C}[u, v]^{\otimes l}$.

Definition 5.13. Let $\mu \in \mathfrak{h}^{*}$ be a dominant integral weight, and let $W_{G}(\mu)$ be the maximal finite-dimensional module over $\mathfrak{s l}_{n}(\mathbb{C}[u, v])$ generated by a vector $v_{\mu}$ such that $\left(\mathfrak{n}^{+} \otimes \mathbb{C}[u, v]\right) v_{\mu}=0$ and

$$
(h \otimes P) v_{\mu}=\mu(h) P(0,0) v_{\mu} \quad \text { for } h \in \mathfrak{h} \text { and } P \in \mathbb{C}[u, v]^{G} .
$$

We say that $W_{G}(\mu)$ is the Weyl module for $\mathfrak{s l}_{n}(\mathbb{C}[u, v])$ associated to the ideal $\left(\mathbb{C}[u, v]_{+}^{G}\right)$.

Remark 5.14. The existence of a maximal finite-dimensional module with this property can be proved as in [Feigin and Loktev 2004].

Proposition 5.15. The Weyl module $W_{G}\left(l \omega_{1}\right)$ is Schur-Weyl dual to $H_{G, l}$, that is, $W_{G}\left(l \omega_{1}\right)=\mathrm{SW}_{l}^{n}\left(H_{G, l}\right)$.

Proof. The argument is the same as the one used in [Feigin and Loktev 2004], so we just sketch it. The Weyl module $W_{G}\left(l \omega_{1}\right)$ is the quotient of the global Weyl module of $\mathfrak{s l}_{n}(\mathbb{C}[u, v])$ for the weight $l \omega_{1}$ by the submodule generated by $\left(h \otimes \mathbb{C}[u, v]_{+}^{G}\right) v_{l \omega_{1}}$. The global Weyl module for this weight is $\operatorname{Sym}^{l}\left(\mathbb{C}^{n} \otimes \mathbb{C}[u, v]\right)$, 
and we must quotient by the submodule generated by the action of $\operatorname{Sym}^{l}\left(\mathbb{C}[u, v]_{+}^{G}\right)$. Thus the Weyl module $W_{G}\left(l \omega_{1}\right)$ is obtained by applying the Schur-Weyl construction to the quotient of $\mathbb{C}\left[u_{1}, \ldots, u_{l}, v_{1}, \ldots, v_{l}\right]$ by the ideal generated by the $S_{l} \ltimes G^{\times l}$-invariant polynomials.

The following theorem of R. Vale is a generalization of a theorem of I. Gordon [2003] for $S_{l}$.

Theorem 5.16 [Vale 2007]. The representation $H_{\Gamma, l}$ has a quotient $H_{\Gamma, l}^{0}$ such that the trace on $H_{\Gamma, l}^{0} \otimes$ Sign of a permutation $\sigma \in S_{l}$ consisting of s cycles is equal to $(d l+1)^{s}$.

Now let us apply Theorem 5.16 to the character calculation for $W_{\Gamma}\left(l \omega_{1}\right)$. Let $F(l, k)$ be the set of functions from $\{1, \ldots, l\}$ to $\{1, \ldots, k\}$. This set admits an action of $S_{l}$ by permutation of the arguments. Denote by $\mathbb{C} F(l, k)$ the corresponding complex representation of $S_{l}$.

Lemma 5.17. Suppose that $\sigma \in S_{l}$ is a product of $s$ cycles. Then the trace of $\sigma$ on $\mathbb{C} F(l, k)$ is equal to $k^{s}$.

Proof. The trace of $\sigma$ is equal to the number of functions stable under the action of $\sigma$. A function is stable if it has the same value on all the elements of each cycle, so it is determined by $s$ elements of $\{1, \ldots, k\}$.

Lemma 5.18. The $\mathfrak{s l}_{n}$-module $\mathrm{SW}_{l}^{n}(\mathbb{C} F(l, k) \otimes \operatorname{Sign})$ is isomorphic to $\bigwedge^{l}\left(\left(\mathbb{C}^{n}\right)^{\oplus k}\right)$. Proof. Note that $\left(\mathbb{C}^{n}\right)^{\otimes l} \otimes \mathbb{C} F(l, k)$ is isomorphic to $\left(\left(\mathbb{C}^{n}\right)^{\oplus k}\right)^{\otimes l}$ as an $\mathrm{SL}_{n} \times S_{l^{-}}$ module. The isomorphism can be constructed as the map sending $\left(v_{1} \otimes \cdots \otimes v_{l}\right) \otimes$ $f$ to $v_{1}^{(f(1))} \otimes \cdots \otimes v_{l}^{(f(l))}$, where $v^{(i)}$ belongs to the $i$-th summand of $\left(\mathbb{C}^{n}\right)^{\oplus k}$. Then the lemma follows by restricting this isomorphism to the Sign component.

Theorem 5.19. The Weyl module $W_{\Gamma}\left(l \omega_{1}\right)$ has a quotient that, as a representation of $\mathfrak{s l}_{n}$, is isomorphic to $\bigwedge^{l}\left(\left(\mathbb{C}^{n}\right)^{\oplus(d l+1)}\right)$.

Proof. Since $H_{\Gamma, l}^{0}$ is a quotient of $H_{\Gamma, l}$, we have by Proposition 5.15 that $\mathrm{SW}_{l}^{n}\left(H_{\Gamma, l}^{0}\right)$ is a quotient of $W_{\Gamma}\left(l \omega_{1}\right)$. Then Lemma 5.17 and Theorem 5.16 imply that $H_{\Gamma, l}^{0}$ is isomorphic to $\mathbb{C} F(l, k) \otimes \operatorname{Sign}$ with $k=d l+1$. By Lemma 5.18, $\operatorname{SW}_{l}^{n}\left(H_{\Gamma, l}^{0}\right)$ is thus equal to $\bigwedge^{l}\left(\left(\mathbb{C}^{n}\right)^{\oplus(d l+1)}\right)$.

Corollary 5.20. The dimension of $W_{\Gamma}\left(l \omega_{1}\right)$ is bounded below by $\left(\begin{array}{c}n(d l+1) \\ l\end{array}\right)$.

By modifying slightly the argument in the previous paragraphs, we can give a lower bound also for some local Weyl modules when $\mathscr{A}=\mathbb{C}[u, v]^{\Gamma}$. Let us introduce an action of $\Gamma^{\times l}$ on $\mathbb{C} F(l, k)$ : If we fix a generator $\xi_{i}$ of the $i$-th copy of $\Gamma$ in $\Gamma^{\times l}$ and if $f \in \mathbb{C} F(l, k)$, then $\xi_{i}(f)=\zeta^{j-1} f$ if $f(i)=j$. This action can be combined with the one associated to $S_{l}$ to obtain an action of $\Gamma^{\times l} \rtimes S_{l}$. The trace of $\xi_{i}$ on $\mathbb{C} F(l, k)$ is $\left(\sum_{j=0}^{k-1} \zeta^{j}\right) k^{l-1}$, which equals $\left(\sum_{j=0}^{\bar{k}} \zeta^{j}\right) k^{l-1}$, where $0 \leq \bar{k} \leq d-1$ and $k-1 \equiv \bar{k} \bmod (d)$. In particular, if $k \equiv 1 \bmod (d)$, then this trace is $k^{l-1}$. 
Theorem 5.16 (see [Vale 2007]) also states that the trace of $\xi_{i}$ on $H_{\Gamma, l}^{0} \otimes$ Sign is equal to $(d l+1)^{l-1}$. That theorem actually applies to any element in $\Gamma^{\times l} \rtimes S_{l}$, and from it we can deduce that we have an isomorphism of $\Gamma^{\times l} \rtimes S_{l}$-modules between $H_{\Gamma, l}^{0}$ and $\mathbb{C} F(l, l d+1) \otimes$ Sign. $\left(\Gamma^{\times l}\right.$ acts trivially on Sign.) Therefore,

$$
\left(H_{\Gamma, l}^{0}\right)^{\Gamma^{\times l}} \cong \mathbb{C} F(l, l+1) \otimes \operatorname{Sign}
$$

since the functions in $\mathbb{C} F(l, l d+1)$ that are invariants under $\Gamma^{\times l}$ can be identified with $\mathbb{C} F(l, l+1)$.

Now, we can repeat the argument we used above. $\operatorname{SW}_{l}^{n}\left(\left(H_{\Gamma, l}^{0}\right)^{\Gamma^{\times l}}\right)$ is a quotient of $\operatorname{SW}_{l}^{n}\left(H_{\Gamma, l}^{\Gamma^{\times l}}\right)$ isomorphic to $\bigwedge^{l}\left(\left(\mathbb{C}^{n}\right)^{\oplus(l+1)}\right)$. Let $W^{\Gamma}(\mu)$ be defined as $W_{\Gamma}(\mu)$ in Definition 5.13, but with $\mathbb{C}[u, v]$ replaced by $\mathbb{C}[u, v]^{\Gamma}$. The Weyl module $W^{\Gamma}\left(l \omega_{1}\right)$ is isomorphic to $\mathrm{SW}_{l}^{n}\left(\mathrm{DH}_{l}\left(\mathbb{C}[u, v]^{\Gamma}\right)\right)$. Since $\mathrm{DH}_{l}\left(\mathbb{C}[u, v]^{\Gamma}\right) \cong\left(H_{\Gamma, l}\right)^{\Gamma^{\times l}}$, we conclude that $W^{\Gamma}\left(l \omega_{1}\right)$ has a quotient isomorphic to $\bigwedge^{l}\left(\left(\mathbb{C}^{n}\right)^{\oplus(l+1)}\right)$ as an $\mathfrak{s l}_{n}$-module, whence the following corollary.

Corollary 5.21. The dimension of $W^{\Gamma}\left(l \omega_{1}\right)$ is bounded below by $\left(\begin{array}{c}n(l+1) \\ l\end{array}\right)$.

There is no reason to expect that the lower bound in Corollary 5.20 is the best possible. Indeed, we can show that it is too low when $d=2$ and $l=4$ by following ideas of I. Gordon. In this case, $\Gamma^{\times l} \rtimes S_{l}$ is isomorphic to the Weyl group $W$ of type $B_{4}$. M. Haiman [1994] explains that the ring of diagonal coinvariants in this case has dimension $9^{4}+1$, which is one more than the dimension of a certain quotient introduced in [Haiman 1994, Conjectures 7.1.2, 7.1.3 and 7.2.3]. These conjectures were proved by I. Gordon [2003] and we denoted above this quotient by $H_{\Gamma, l}^{0}$.

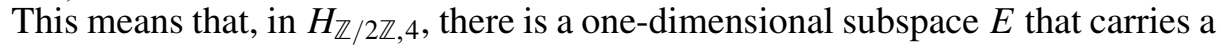
nontrivial representation of $W$. There is an action of $\mathfrak{s l}_{2}(\mathbb{C})$ on $H_{\mathbb{Z} / 2 \mathbb{Z}, 4}$ commuting with the action of $S_{4}$ (this is actually true in $\mathbb{C}\left[u_{1}, v_{1}, \ldots, u_{l}, v_{l}\right]$ for any $l \in \mathbb{Z}_{\geq 1}$ ), so $E$ is also a representation of $\mathfrak{s l}_{2}(\mathbb{C})$ and must thus be trivial. The standard diagonal element $h \in \mathfrak{s l}_{2}(\mathbb{C})$ acts by $\sum_{i=1}^{4}\left(u_{i} d / d u_{i}-v_{i} d / d v_{i}\right)$, so this operator acts trivially on $E$, which implies that the monomials that appear in $E$ have their $u$-degree equal to their $v$-degree.

The Weyl module $W_{\mathbb{Z} / 2 \mathbb{Z}}\left(4 \omega_{1}\right)$ is obtained by applying the Schur-Weyl functor

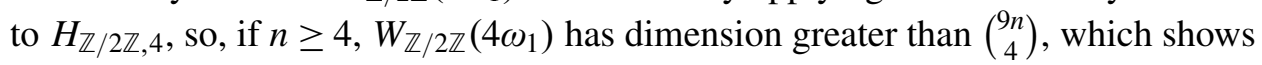
that the lower bound in Corollary 5.20 is too low.

\section{Matrix Lie algebras over rational Cherednik algebras of rank one}

The polynomial ring $\mathbb{C}[u, v]$ can be deformed into the first Weyl algebra $A_{1}=$ $\mathbb{C}\langle u, v\rangle /(v u-u v-1)$, which itself can be viewed as the ring $\mathscr{D}(\mathbb{C})$ of algebraic differential operators on the affine line $\mathbb{A}_{\mathbb{C}}^{1}$. Such differential operators play an important role in the representation theory of Cherednik algebras, and the $G$-DDCA 
of [Guay 2005; 2007; 2009b] are also deformations of the enveloping algebra of $\mathfrak{g l}_{n}\left(A_{1} \rtimes \Gamma\right)$ when $G$ is a finite subgroup of $\mathrm{SL}_{2}(\mathbb{C})$.

More generally, the rational Cherednik algebra $\mathrm{H}_{t, \mathbf{c}}\left(G_{l}\right)$ for the wreath product $G_{l}=G^{\times l} \rtimes S_{l}$ admits two specializations of particular interest:

$$
\mathrm{H}_{t=0, \mathbf{c}=\mathbf{0}}\left(G_{l}\right) \cong \mathbb{C}\left[x_{1}, y_{1}, \ldots, x_{l}, y_{l}\right] \rtimes G_{l} \quad \text { and } \quad \mathrm{H}_{t=1, \mathbf{c}=\mathbf{0}}\left(G_{l}\right) \cong A_{l} \rtimes G_{l},
$$

where $A_{l}$ is the $l$-th Weyl algebra. The representation theories of these two algebras differ greatly. For instance, in the first case, $\mathrm{H}_{t=0, \mathbf{c}=\mathbf{0}}\left(G_{l}\right)$ has infinitely many irreducible finite-dimensional representations, whereas $\mathrm{H}_{t=1, \mathbf{c}=0}\left(G_{l}\right)$ has none. Actually, $\mathrm{H}_{t=1, \mathrm{c}}\left(G_{l}\right)$ does not have any finite-dimensional representations for generic values of $\mathbf{c}$. The $\Gamma$-DDCA also admit two such specializations, and it is reasonable to expect that their representation theories will thus differ noticeably. In this article, we want to start investigating the categories of modules for these two specializations, so, in this section we will study matrix Lie algebras over rational Cherednik algebras of rank one with $t \neq 0$.

Definition 6.1. Let $\mathbf{c}=\left(c_{1}, \ldots, c_{d-1}\right) \in \mathbb{C}^{d-1}$. The rational Cherednik algebra $\mathrm{H}_{t, \mathbf{c}}(\Gamma)$ of rank one is the algebra generated by elements $u, v, \gamma$ with $\gamma \in \Gamma=\mathbb{Z} / d \mathbb{Z}$ and the relations $\gamma u \gamma^{-1}=\zeta u, \gamma v \gamma^{-1}=\zeta^{-1} v$ and

$$
v u-u v=t+\sum_{i=1}^{d-1} c_{i} \xi^{i}, \quad \text { where } \xi \text { is a generator of } \Gamma .
$$

It will be convenient to rewrite (6) in the form $v u-u v=t+\sum_{i=0}^{d-1} \tilde{c}_{i}\left(\mathbf{e}_{i}-\mathbf{e}_{i+1}\right)$ for some $\tilde{c}_{i} \in \mathbb{C}$. We will need to use later the element $\omega$ that can be written in the three equivalent ways

$$
\begin{aligned}
\omega & =-u v+\sum_{i=0}^{d-1} \tilde{c}_{i} \mathbf{e}_{i+1}=-v u+t+\sum_{i=0}^{d-1} \tilde{c}_{i} \mathbf{e}_{i} \\
& =-\frac{u v+v u}{2}+\frac{t}{2}+\frac{1}{2} \sum_{i=0}^{d-1} \tilde{c}_{i}\left(\mathbf{e}_{i}+\mathbf{e}_{i+1}\right) .
\end{aligned}
$$

Then one can check that $[\omega, u]=-t u$ and $[\omega, v]=t v$.

Definition 6.2. Let $\mathbf{c}=\left(c_{1}, \ldots, c_{d-1}\right) \in \mathbb{C}^{d-1}$. We will call the trigonometric Cherednik algebra of rank one the algebra $\mathbf{H}_{t, \mathbf{c}}(\Gamma)=\mathbb{C}\left[u^{ \pm 1}\right] \otimes \mathbb{C}[u] \mathrm{H}_{t, \mathbf{c}}(\Gamma)$.

Remark 6.3. Trigonometric Cherednik algebras exist only for Weyl groups (real Coxeter groups), but we propose to use the terminology in the previous definition because it is convenient. Moreover, as is explained in [Guay 2009b], $\mathbf{H}_{t, \mathbf{c}}(\Gamma)$ depends actually only on the $t$ parameter, that is, $\mathbf{H}_{t, \mathbf{c}}(\Gamma) \cong \mathbf{H}_{t, \mathbf{c}=\mathbf{0}}(\Gamma)$, but this is not 
true for $\mathrm{H}_{t, \mathbf{c}}(\Gamma)$. An explicit isomorphism $\mathbf{H}_{t, \mathbf{c}}(\Gamma) \stackrel{\sim}{\longrightarrow} \mathbf{H}_{t, \mathbf{c}=\mathbf{0}}(\Gamma)$ is given by

$$
v \mapsto v+\left(\sum_{i=1}^{d-1} \frac{c_{i}}{1-\zeta^{-i}} \xi^{i}\right) u^{-1} .
$$

Note also that $\mathbf{H}_{t, \mathbf{c}}(\Gamma)$ is generated by $\omega, u, u^{-1}, \Gamma$ and that $\left[\omega, u^{-1}\right]=t u^{-1}$.

The associative algebras $\mathbf{H}_{t, \mathbf{c}}(\Gamma)$ and $\mathrm{H}_{t, \mathbf{c}}(\Gamma)$ can be turned into Lie algebras in the usual way, and the representation theory of a central extension of the Lie algebra $\mathfrak{g l}_{n}\left(\mathbf{A}_{1}\right)$ was studied in [Boyallian et al. 1998; Kac and Radul 1993]. (Here, $\mathbf{A}_{1}$ is the algebra of differential operators on $\mathbb{C}^{\times}$.) Boyallian and Liberati [2002] considered the case of the quantum torus $\mathscr{D}_{q}\left(\mathbb{C}^{\times}\right)=\mathbb{C}\left\langle u^{ \pm 1}, v^{ \pm 1}\right\rangle /(v u=q u v)$. In this section, we present some results about the structure of the Lie algebras $\mathfrak{s l}_{n}\left(\mathrm{H}_{t, \mathbf{c}}(\Gamma)\right)$ and $\mathfrak{s l}_{n}\left(\mathbf{H}_{t, \mathbf{c}}(\Gamma)\right)$, mostly when $t \neq 0$.

The (Lie) algebras $\mathbf{H}_{t, \mathbf{c}}(\Gamma)$ and $\mathrm{H}_{t, \mathbf{c}}(\Gamma)$ are graded as $\operatorname{deg}(u)=-1, \operatorname{deg}(v)=1$ and $\operatorname{deg}(\gamma)=0$. This induces gradings on the associative algebras $M_{n}\left(\mathbf{H}_{t, \mathbf{c}}(\Gamma)\right)$ and $M_{n}\left(\mathrm{H}_{t, \mathbf{c}}(\Gamma)\right)$ and on the Lie algebras $\mathfrak{g l}_{n}\left(\mathbf{H}_{t, \mathbf{c}}(\Gamma)\right)$ and $\mathfrak{g l}_{n}\left(\mathrm{H}_{t, \mathbf{c}}(\Gamma)\right)$. However, we will consider instead the grading

$$
\operatorname{deg}\left(E_{i j} v^{r} u^{s} \gamma\right)=(r-s) n+j-i .
$$

In the case $\mathbf{H}_{t=1, \mathbf{c}=\mathbf{0}}(\Gamma=\{1\})$, this is the opposite of the principal $\mathbb{Z}$-gradation considered in [Boyallian et al. 1998]. The graded pieces of degree $k$ will be denoted $\mathfrak{g l}_{n}\left(\mathbf{H}_{t, \mathbf{c}}(\Gamma)\right)[k]$ and $\mathfrak{g l}_{n}\left(\mathrm{H}_{t, \mathbf{c}}(\Gamma)\right)[k]$.

6A. Central extensions. It was found in [Alev et al. 2000] that $H H_{1}\left(A_{1} \rtimes \Gamma\right)=0$; hence $H C_{1}\left(A_{1} \rtimes \Gamma\right)=0$. Furthermore, it is proved in [Etingof and Ginzburg 2002] for any $\mathbf{c}$ that $H H_{1}\left(\mathrm{H}_{t, \mathbf{c}}(\Gamma)\right)=0$ for all $t \in \mathbb{C}^{\times}$except in a countable set. For all such values of $t$ and $\mathbf{c}$, the Lie algebra $\mathfrak{s l}_{n}\left(\mathrm{H}_{t, \mathbf{c}}(\Gamma)\right)$ has no nontrivial central extension. For this reason, contrary to [Boyallian et al. 1998], we will not consider central extensions.

6B. Parabolic subalgebras. In this subsection, we will assume that $t \neq 0$, so, without loss of generality, let us set $t=1$. For a Lie algebra with triangular decomposition, one usually wants to construct representations by induction from its nonnegative Lie subalgebra (a sort of Borel subalgebra) or, more generally, from a bigger subalgebra that contains this one. This suggests that the following definition may be relevant.

Definition 6.4. [Boyallian et al. 1998] A parabolic subalgebra $\mathfrak{q}$ of the Lie algebra $\mathfrak{g l}_{n}\left(\mathrm{H}_{t=1, \mathbf{c}}(\Gamma)\right)$ is a graded Lie subalgebra of the form

$$
\begin{array}{lll}
\mathfrak{q}=\bigoplus_{\mathbb{Z}} \mathfrak{q}[k], & \mathfrak{q}[k]=\mathfrak{g l}_{n}\left(\mathrm{H}_{t=1, \mathbf{c}}(\Gamma)\right)[k] & \text { if } k \geq 0, \\
& \mathfrak{q}[k] \subset \mathfrak{g l}_{n}\left(\mathrm{H}_{t=1, \mathfrak{c}}(\Gamma)\right)[k] & \text { if } k<0 .
\end{array}
$$


For $k<0$, we can decompose $\mathfrak{q}[k]$ as

$$
\mathfrak{q}[k]=\bigoplus_{\substack{r, l, i, j \\-r n+j-i=k}} E_{i j} u^{r} I_{k}^{i, l} \mathbf{e}_{l} \quad \text { for some subspace } I_{k}^{i, l} \subset \mathbb{C}[\omega] .
$$

Lemma 6.5. The subspace $I_{k}^{i, l}$ is an ideal of $\mathbb{C}[\omega]$.

Proof. Let $E_{i j} u^{r} p(\omega) \mathbf{e}_{l} \in \mathfrak{q}[k]$ with $p(\omega) \in I_{k}^{i, l}$, and choose $f(\omega) \in \mathbb{C}[\omega]$. If $r=0$, then, since $E_{i i} f(\omega) \mathbf{e}_{l} \in \mathfrak{q}[0]$, we deduce that $\left[E_{i i} f(\omega) \mathbf{e}_{l}, E_{i j} p(\omega) \mathbf{e}_{l}\right]=$ $E_{i j} f(\omega) p(\omega) \mathbf{e}_{l} \in \mathfrak{q}[k]$ if $1 \leq i \neq j \leq n$; hence $f(\omega) p(\omega) \in I_{k}^{i, l}$.

Now suppose that $r>0$. We want to prove by induction on $a \in \mathbb{Z}_{\geq 0}$ that $\omega^{a} p(\omega) \in I_{k}^{i, l}$. We note that

$$
\left[I \omega^{a+1}, E_{i j}\left(u^{r} p(\omega) \mathbf{e}_{l}\right)\right]=E_{i j}\left(u^{r}\left((\omega-r)^{a+1}-\omega^{a+1}\right) p(\omega) \mathbf{e}_{l}\right) \in \mathfrak{q}[k]
$$

and that the term of highest power in $(\omega-r)^{a+1}-\omega^{a+1}$ is $-r(a+1) \omega^{a}$, so that we can apply induction.

Following the ideas of [Boyallian et al. 1998; Kac and Radul 1993], we choose a monic generator $b_{k}^{i, l}(\omega)$ of the principal ideal $I_{k}^{i, l}$ if this ideal is nonzero; otherwise, we set $b_{k}^{i, l}(\omega)=0$. These are called the characteristic polynomials of $\mathfrak{q}$.

Definition 6.6. A parabolic subalgebra $\mathfrak{q}$ is nondegenerate if $\mathfrak{q}[k] \neq 0$ for all $k \in \mathbb{Z}$.

Proposition 6.7. A parabolic subalgebra $\mathfrak{q}$ is nondegenerate if and only if the polynomials $b_{-1}^{i, l}(\omega)$ are all nonzero for $1 \leq i \leq n$ and $0 \leq l \leq d-1$.

Proof. The parabolic subalgebra $\mathfrak{q}$ is nondegenerate if and only if the polynomials $b_{k}^{i, l}(\omega)$ for $1 \leq i \leq n$ and $0 \leq l \leq d-1$ are all nonzero for all $k \in \mathbb{Z}_{\leq-1}$, so it is enough to prove for $i=1, \ldots, n$ that if $b_{k}^{i, l}(\omega)$ and $b_{-1}^{i-1, l}(\omega)$ are nonzero, then $b_{k-1}^{i, l}(\omega)$ is also nonzero and divides $b_{k}^{i-1, l}(\omega) b_{-1}^{i, l+r}(\omega-r)$. Here, if $2 \leq i \leq n$, then $r$ is determined by $k+i-1=-r n+j$ for some $1 \leq j \leq n$. (We set $b_{k}^{0, l}(\omega)=b_{k}^{n, l}(\omega)$.)

If $i \neq 1$, we have

$$
\begin{aligned}
& {\left[E_{i, i-1} b_{-1}^{i, l+r}(\omega) \mathbf{e}_{l+r}, E_{i-1, j} u^{r} b_{k}^{i-1, l}(\omega) \mathbf{e}_{l}\right]} \\
& \quad=E_{i j} u^{r} b_{-1}^{i, l+r}(\omega-r) b_{k}^{i-1, l}(\omega) \mathbf{e}_{l}-\delta_{j, i} \delta_{r 0} E_{i-1, i-1} u^{r} b_{-1}^{i, l+r}(\omega) b_{k}^{i-1, l}(\omega) \mathbf{e}_{l+r} \\
& \quad \in[\mathfrak{q}[-1], \mathfrak{q}[k]]
\end{aligned}
$$

and $[\mathfrak{q}[-1], \mathfrak{q}[k]] \subset \mathfrak{q}[k-1]$, so $b_{-1}^{i, l+r}(\omega-r) b_{k}^{i-1, l}(\omega) \in I_{k-1}^{i, l}$ and the claim is true if $i \neq n$.

To prove the claim when $i=1$ (and with $r$ determined by $k=-(r+1) n+j$ ), we consider the commutator

$$
\begin{aligned}
& {\left[E_{1 n} u b_{-1}^{1, l+r}(\omega) \mathbf{e}_{l+r}, E_{n j} u^{r} b_{k}^{n, l}(\omega) \mathbf{e}_{l}\right]} \\
& \quad=E_{1 j} u^{r+1} b_{-1}^{1, l+r}(\omega-r) b_{k}^{n, l}(\omega) \mathbf{e}_{l}-\delta_{j 1} \delta_{r,-1} E_{n n} u^{r+1} b_{k}^{n, l}(\omega-1) b_{-1}^{1, l+r}(\omega) \mathbf{e}_{l+r},
\end{aligned}
$$


which belongs to $[\mathfrak{q}[-1], \mathfrak{q}[k]] \subset \mathfrak{q}[k-1]$, so $b_{-1}^{1, l+r}(\omega-r) b_{k}^{n, l}(\omega) \in I_{k-1}^{1, l}$.

Using similar computations, one can prove for $i=1, \ldots, n$ that if $b_{k-1}^{i, l}(\omega) \neq 0$, then $b_{k}^{i-1, l}(\omega)$ is nonzero and divides $b_{k-1}^{i, l}(\omega)$.

The characteristic polynomials $b_{-1}^{i, l}(\omega)$ for $1 \leq i \leq n$ and $0 \leq l \leq d-1$ can help us describe the derived Lie subalgebra $[\mathfrak{q}, \mathfrak{q}]$. Set

$$
\begin{aligned}
\mathfrak{g l}_{n}\left(\mathrm{H}_{t=1, \mathbf{c}}(\Gamma)\right)[0, \mathbf{b}] & \\
= & \operatorname{span}\left\{H_{i} \omega^{r} b_{-1}^{i+1, l}(\omega) \mathbf{e}_{l} \mid 1 \leq i \leq n-1, r \in \mathbb{Z}_{\geq 0}, 0 \leq l \leq d-1\right\} \\
& \oplus \operatorname{span}\left\{E_{11} u v(\omega+1)^{r} b_{-1}^{1, l}(\omega+1) \mathbf{e}_{l+1}-E_{n n} v u \omega^{r} b_{-1}^{1, l}(\omega) \mathbf{e}_{l} \mid r \in \mathbb{Z}_{\geq 0}\right\} .
\end{aligned}
$$

Proposition 6.8. Let $\mathbf{b}=\left(b_{-1}^{i, l}(\omega)\right)_{1 \leq i \leq n}^{0 \leq l \leq d-1}$ be the first $n d$ characteristic polynomials of the parabolic subalgebra $\mathfrak{q}$. Then

$$
[\mathfrak{q}, \mathfrak{q}]=\left(\bigoplus_{k \in \mathbb{Z}, k \neq 0} \mathfrak{q}[k]\right) \oplus \mathfrak{g l}_{n}\left(\mathrm{H}_{t=1, \mathbf{c}}(\Gamma)\right)[0, \mathbf{b}] .
$$

Proof. Since $\mathfrak{q}[k+1]=[\mathfrak{q}[k], \mathfrak{q}[1]]$ if $k \in \mathbb{Z}_{\geq 0}$, it suffices to show that $[\mathfrak{q}[1], \mathfrak{q}[-1]]=$ $\mathfrak{g l}_{n}\left(\mathrm{H}_{t=1, \mathbf{c}}(\Gamma)\right)[0, \mathbf{b}]$. We compute

$$
\begin{array}{rlrl}
{\left[E_{i, i+1} \omega^{r} \mathbf{e}_{l_{1}}, E_{i+1, i} b_{-1}^{i+1, l_{2}}(\omega) \mathbf{e}_{l_{2}}\right]} & =\delta_{l_{1} l_{2}}\left(E_{i i}-E_{i+1, i+1}\right) \omega^{r} b_{-1}^{i+1, l_{2}}(\omega) \mathbf{e}_{l_{1}}, \\
{\left[E_{1 n} u b_{-1}^{1, l_{1}}(\omega) \mathbf{e}_{l_{1}}, E_{n 1} v(\omega+1)^{r} \mathbf{e}_{l_{2}}\right]} & =\delta_{l_{1}+1, l_{2}}\left(E_{11} u v(\omega+1)^{r} b_{-1}^{1, l_{1}}(\omega+1) \mathbf{e}_{l_{2}}\right. \\
{\left[E_{i, i+1} \omega^{r} \mathbf{e}_{l_{1}}, E_{1 n} u b_{-1}^{1, l_{2}}(\omega) \mathbf{e}_{l_{2}}\right]} & =0, & \left.-E_{n n} v u \omega^{r} b_{-1}^{1, l_{1}}(\omega) \mathbf{e}_{l_{1}}\right), \\
{\left[E_{i+1, i} b_{-1}^{i+1, l_{1}}(\omega) \mathbf{e}_{l_{1}}, E_{n 1} v \omega^{r} \mathbf{e}_{l_{2}}\right]} & =0 & \text { if } 1 \leq i \leq n-1 .
\end{array}
$$

6C. Embedding into $\overline{\mathfrak{g l}}_{\infty}$. One of the main objects used to study the representation theory of $\mathfrak{g l}_{n}\left(\mathbf{A}_{1}\right)$ in [Boyallian et al. 1998; Kac and Radul 1993] is an embedding of the algebra $M_{n}\left(\mathbf{A}_{1}\right)$ into the algebra $\bar{M}_{\infty}$ of infinite matrices with only finitely many nonzero diagonals. This induces an embedding of the Lie algebra $\mathfrak{g l}_{n}\left(\mathbf{A}_{1}\right)$ into $\overline{\mathfrak{g l}}_{\infty}$. It comes from the action of $\mathfrak{g l}_{n}\left(\mathbf{A}_{1}\right)$ on $\mathbb{C}^{n} \otimes \mathbb{C}\left[u, u^{-1}\right]$. In this subsection, we obtain similar embeddings for $\mathfrak{g l}_{n}\left(\mathrm{H}_{t=1, \mathbf{c}}(\Gamma)\right)$ and $\mathfrak{g l}_{n}\left(\mathbf{A}_{1} \rtimes \Gamma\right)$ when $\Gamma$ is cyclic. The embedding $\mathfrak{g l}_{n}\left(\mathbf{A}_{1} \rtimes \Gamma\right) \hookrightarrow \overline{\mathfrak{g l}}_{\infty}$ is the same as the one considered in the two papers above when $\Gamma$ is trivial, and $\mathfrak{g l}_{n}\left(\mathrm{H}_{t=1, \mathbf{c}}(\Gamma)\right) \hookrightarrow \overline{\mathfrak{g l}}_{\infty}$ comes also from the action of $\mathfrak{g l}_{n}\left(\mathrm{H}_{t=1, \mathrm{c}}(\Gamma)\right)$ on $\mathbb{C}^{n} \otimes \mathbb{C}\left[u, u^{-1}\right]$ via the Dunkl embedding of $\mathrm{H}_{t=1, \mathbf{c}}(\Gamma)$. (We will reserve the notation $M_{\infty}$ and $\mathfrak{g l}_{\infty}$ for the algebra and the Lie algebra of infinite matrices with finitely many nonzero entries.)

The space $M_{\infty}$ has a linear basis of elementary matrices $E_{i j}$ with $(i, j) \in \mathbb{Z} \times \mathbb{Z}$. The embedding of associative algebras $\imath: M_{n}\left(\mathbf{A}_{1} \rtimes \Gamma\right) \hookrightarrow \bar{M}_{\infty}$ is given explicitly 
by the formula

${ }_{l}\left(E_{i j} u^{s} \omega^{r} \mathbf{e}_{k}\right)=\sum_{l \in \mathbb{Z}}(-l d-k)^{r} E_{(l d+k+s) n+i-1,(l d+k) n+j-1} \quad$ for $s \in \mathbb{Z}, 0 \leq k \leq d-1$.

This restricts to an embedding $\imath: M_{n}\left(\mathrm{H}_{t=1, \mathbf{c}}(\Gamma)\right) \hookrightarrow \bar{M}_{\infty}$ by pulling back via $\mathrm{H}_{t=1, \mathbf{c}}(\Gamma) \hookrightarrow \mathbf{H}_{t=1, \mathbf{c}}(\Gamma) \cong \mathbf{A}_{1} \rtimes \Gamma$. Explicitly, since

$$
v=-u^{-1}\left(\omega-\sum_{l=0}^{d-1} \tilde{c}_{l-1} \mathbf{e}_{l}\right),
$$

we get

$$
l\left(E_{i j} v \mathbf{e}_{k}\right)=\sum_{l \in \mathbb{Z}}\left(l d+k+\tilde{c}_{k-1}\right) E_{(l d+k-1) n+i-1,(l d+k) n+j-1} .
$$

This can be extended to

(7) $\quad l\left(E_{i j} v^{s} \omega^{r} \mathbf{e}_{k}\right)$

$$
=\sum_{l \in \mathbb{Z}}\left(\prod_{p=0}^{s-1}\left(l d+k-p+\tilde{c}_{k-p-1}\right)\right)(-l d-k)^{r} E_{(l d+k-s) n+i-1,(l d+k) n+j-1} .
$$

The principal grading on the algebra $\bar{M}_{\infty}$ and on the Lie algebra $\overline{\mathfrak{g l}}_{\infty}$ is given by $\operatorname{deg}\left(E_{i j}\right)=j-i$, and the embedding $\imath$ respects all the gradings.

We will need, as in [Boyallian et al. 1998], to consider infinite matrices over the ring of truncated polynomials $R_{m}=\mathbb{C}[t] /\left(t^{m+1}\right)$. Fixing $a \in \mathbb{C}$, we define an algebra map $\varphi_{a}^{[m]}: M_{n}\left(\mathrm{H}_{t=1, \mathrm{c}}(\Gamma)\right) \rightarrow \bar{M}_{\infty}\left(R_{m}\right)$ by

$$
\begin{aligned}
\varphi_{a}^{[m]}\left(E_{i j} u \mathbf{e}_{k}\right) & =\sum_{l \in \mathbb{Z}} E_{(l d+k+1) n+i-1,(l d+k) n+j-1}, \\
\varphi_{a}^{[m]}\left(E_{i j} v \mathbf{e}_{k}\right) & =\sum_{l \in \mathbb{Z}}\left(l d+k+a+t+\tilde{c}_{k-1}\right) E_{(l d+k-1) n+i-1,(l d+k) n+j-1} .
\end{aligned}
$$

This extends to a map $\varphi_{a}^{[m]}: M_{n}\left(\mathbf{A}_{1} \rtimes \Gamma\right) \rightarrow \bar{M}_{\infty}\left(R_{m}\right)$. Explicitly,

$$
\varphi_{a}^{[m]}\left(E_{i j} u^{s} \omega^{r} \mathbf{e}_{k}\right)=\sum_{l \in \mathbb{Z}}(-l d-k-a-t)^{r} E_{(l d+k+s) n+i-1,(l d+k) n+j-1}
$$

for $s \in \mathbb{Z}$ and $0 \leq k \leq d-1$.

This embedding when $d=2$ and $n=1$ is related to the embedding considered in [Shoikhet 1998] from the Lie algebra $\mathfrak{g l}_{\lambda}$ to $\overline{\mathfrak{g l}}_{\infty, s}$ (in the notation of that paper) since $\mathfrak{g l}_{\lambda}$ is obtained by turning into a Lie algebra a certain primitive quotient of $\mathfrak{U}_{\mathfrak{s}} \mathfrak{L}_{2}(\mathbb{C})$ and this primitive quotient is isomorphic to the spherical subalgebra of $\mathrm{H}_{t=1, c=\lambda}(\mathbb{Z} / 2 \mathbb{Z})$. 
6D. Geometric interpretation. Kac and Radul [1993] observed that the algebra of holomorphic differential operators on $\mathbb{C}^{\times}$has a geometric interpretation in terms of a certain infinite-dimensional vector bundle over the cylinder $\mathbb{C} / \mathbb{Z}$. The algebras $\mathbf{A}_{1} \rtimes \Gamma$ and $A_{1} \rtimes \Gamma$ afford similar interpretations. To explain it, we have to extend them to a holomorphic setting.

Let $O(w)$ be the ring of entire functions (holomorphic on all of $\mathbb{C}$ ) in the variable $w$. Let $\mathbf{A}_{1}^{\mathbb{O}} \rtimes \Gamma$ to be the span of the operators of the form $u^{r} f(w) \gamma$ with $f \in \mathcal{O}(w)$ and $r \in \mathbb{Z}$. This span has an algebra structure extending the one on $\mathbf{A}_{1} \rtimes \Gamma$. Let $A_{1}^{\mathbb{O}} \rtimes \Gamma$ be the subalgebra of $\mathbf{A}_{1}^{\mathbb{O}} \rtimes \Gamma$ consisting of linear combinations of operators of the form $u^{r} f(w) \gamma$ and $v^{s} f(w) \gamma$ with $r, s \geq 0$ and $f$ holomorphic.

For $k \in \mathbb{Z}$, we define an automorphism $\theta_{k}$ of $\bar{M}_{\infty}$ and of $\overline{\mathfrak{g l}}_{\infty}$ by $\theta_{k}\left(E_{i j}\right)=$ $E_{i+k, j+k}$. For $0 \leq k \leq d-1$, let $\bar{M}_{\infty}^{k} \subset \bar{M}_{\infty}$ be the subspace of matrices such that the $(i, j)$ entry is zero if $j \notin \bigcup_{l \in \mathbb{Z}}[l d n+k n, l d n+(k+1) n$ [. The following definition is adapted from [Kac and Radul 1993, Definition 3.4].

Definition 6.9. An $(n, d)$-monodromic loop is a holomorphic map $\ell: \mathbb{C} \rightarrow \bar{M}_{\infty}$ such that $\ell(w)=\ell_{0}(w)+\cdots+\ell_{d-1}(w)$ with $\ell_{k}(w-d)=\theta_{n}^{d} \ell_{k}(w)$ and $\ell_{k}(w) \in \bar{M}_{\infty}^{k}$ for $0 \leq k \leq d-1$.

When $d=1$, the following proposition was established in [Kac and Radul 1993].

Proposition 6.10. The algebra $M_{n}\left(\mathbf{A}_{1}^{0} \rtimes \Gamma\right)$ is isomorphic to the algebra $\mathscr{L}_{n, d}$ of $(n, d)$-monodromic loops.

Proof. We can construct a map $M_{n}\left(\mathbf{A}_{1}^{0} \rtimes \Gamma\right) \rightarrow \mathscr{L}_{n, d}$ by $E \mapsto\left(w \mapsto \varphi_{w}^{[0]}(E)\right)$. That the formula $w \mapsto \varphi_{w}^{[0]}(E)$ defines an $(n, d)$-monodromic loop follows from the formula for $\varphi_{w}^{[0]}$. The inverse is given in the following way. If, given a monodromic loop $\ell$, the loop $\ell_{k}$ is concentrated along the $(s n+m)$-th diagonal (for $0 \leq m \leq n-1$ ) below the main one (so $s n+m \geq 0$ - if it is above, the argument is similar), so that $\ell_{k}=\sum_{i=1}^{n} \sum_{l \in \mathbb{Z}} f_{i, l, k}(w) E_{(l d+k+s) n+m+i-1,(l d+k) n+i-1}$, then the preimage of $\ell_{k}$ is $\sum_{i=1}^{n-m} E_{m+i, i} u^{s} f_{i, 0, k}(-\omega-k) \mathbf{e}_{k}+\sum_{i=1}^{m} E_{i, n-m+i} u^{s+1} f_{n-m+i, 0, k}(-\omega-k) \mathbf{e}_{k}$.

Since $M_{n}\left(A_{1}^{\mathbb{O}} \rtimes \Gamma\right) \hookrightarrow M_{n}\left(\mathbf{A}_{1}^{\mathbb{O}} \rtimes \Gamma\right)$, we can identify $M_{n}\left(A_{1}^{\mathbb{O}} \rtimes \Gamma\right)$ with the algebra of $(n, d)$-monodromic loops $\ell$ such that, writing $\ell(w)=\sum_{i, j \in \mathbb{Z}} \ell_{i, j}(w) E_{i j}$, we have that, if $i=l_{1} n+p_{1}-1, j=l_{2} n+p_{2}-1,1 \leq p_{1}, p_{2} \leq n$ and $l_{1}<l_{2}$, then $\ell_{i, j}(w)=0$ for $w=p-l_{2} d$ and $p=0, \ldots, l_{2}-l_{1}-1$.

\section{Highest weight representations for matrix Lie algebras over Cherednik algebras of rank one}

Inspired by the papers [Ginzburg et al. 2003; Guay 2005], we suggest a notion of category 0 for the Lie algebra $\mathfrak{s l}_{n}\left(\mathrm{H}_{t=1, \mathbf{c}}(\Gamma)\right)$, and we study certain modules in it, which we call quasifinite highest weight modules. 
Definition 7.1. Assume that $t \neq 0$. The category $\mathcal{O}\left(\mathfrak{s l}_{n}\left(\mathrm{H}_{t=1, \mathbf{c}}(\Gamma)\right)\right)$ is the category of finitely generated modules $M$ over $\mathfrak{U}_{\mathfrak{s l}} l_{n}\left(\mathrm{H}_{t=1, \mathbf{c}}(\Gamma)\right)$ upon which $\mathfrak{s l}_{n}(v \mathbb{C}[v])$ acts locally nilpotently.

This definition applies also to the $\Gamma$-deformed double current algebras $\mathrm{D}_{\lambda, \mathbf{b}}^{n}(\Gamma)$ of [Guay 2009b]. One justification for it is that the Schur-Weyl functor studied in [Guay 2005; 2009b] sends modules in the category 0 of a rational Cherednik algebra for $\Gamma^{\times l} \rtimes S_{l}$ to a module in $\mathcal{O}\left(\mathrm{D}_{\lambda, \mathbf{b}}^{n}(\Gamma)\right.$ ) (for appropriate values of $\lambda, \mathbf{b}$ ). It is possible, using induction, to construct analogues of Verma modules in this category, and one can ask about the classification of irreducible (integrable) modules in the category $\mathrm{O}\left(\mathfrak{s l}_{n}\left(\mathrm{H}_{t=1, \mathbf{c}}(\Gamma)\right)\right)$. We will not try to answer this question. Instead, we will study certain modules in these categories by following the ideas in [Boyallian et al. 1998; Kac and Radul 1993].

Recall the grading on $\mathfrak{s l}_{n}\left(A_{1} \rtimes \Gamma\right)$ and the embeddings $\varphi_{a}^{[m]}: \mathfrak{s l}_{n}\left(A_{1} \rtimes \Gamma\right) \hookrightarrow$ $\overline{\mathfrak{g l}}_{\infty}\left(R_{m}\right)$. The Lie algebra $\overline{\mathfrak{g l}}_{\infty}$ has an obvious triangular structure compatible with the grading given by $\operatorname{deg}\left(E_{i j}\right)=j-i$, and the embeddings $\varphi_{a}^{[m]}$ respect the grading on the source and target spaces. The following definition comes naturally from the triangular structure.

Definition 7.2. Let $\lambda_{i, k, r} \in \mathbb{C}$ for $1 \leq i \leq n, 0 \leq k \leq d-1$ and $r \in \mathbb{Z}_{\geq 0}$, and let $\lambda \in \mathfrak{g l}_{n}\left(\mathrm{H}_{1, \mathbf{c}}(\Gamma)\right)[0]^{*}$ be given by $\lambda\left(E_{i i} w^{r} \mathbf{e}_{k}\right)=\lambda_{i, k, r}$. Extending $\lambda$ to a onedimensional representation $\mathbb{C}_{\lambda}$ of the Lie algebra $\mathfrak{g l}_{n}\left(\mathrm{H}_{1, \mathbf{c}}(\Gamma)\right)[\geq 0]$ (which is equal to $\left.\bigoplus_{k=0}^{\infty} \mathfrak{g l}_{n}\left(\mathrm{H}_{1, \mathbf{c}}(\Gamma)\right)[k]\right)$ by letting $\mathfrak{g l}_{n}\left(\mathrm{H}_{1, \mathbf{c}}(\Gamma)\right)[k]$ act trivially if $k>0$, we define the Verma module $M(\lambda)$ by

$$
M(\lambda)=\mathfrak{U g l}_{n}\left(\mathrm{H}_{1, \mathbf{c}}(\Gamma)\right) \otimes \mathfrak{U}_{\mathfrak{g l}}\left(\mathrm{H}_{1, \mathfrak{c}}(\Gamma)\right)[\geq 0] \mathbb{C}_{\lambda} .
$$

The following lemma and definition are quite standard.

Lemma 7.3. The Verma module $M(\lambda)$ has a unique irreducible quotient, which we denote by $L(\lambda)$.

Definition 7.4. We call a $\mathfrak{g l}_{n}\left(\mathrm{H}_{1, \mathbf{c}}(\Gamma)\right)$-module a highest weight module of highest weight $\lambda \in \mathfrak{g l}_{n}\left(\mathrm{H}_{1, \mathbf{c}}(\Gamma)\right)[0]^{*}$ if this module is generated by a vector $v$ on which $h \in \mathfrak{g l}_{n}\left(\mathrm{H}_{1, \mathbf{c}}(\Gamma)\right)[0]$ acts by multiplication by $\lambda(h)$ and $\mathfrak{g l}_{n}\left(\mathrm{H}_{1, \mathbf{c}}(\Gamma)\right)[k]$ acts trivially if $k \in \mathbb{Z}_{>0}$. A vector with this last property is said to be singular.

The highest weight vector which generates the Verma module $M(\lambda)$ will be denoted $v_{\lambda}$.

Given a parabolic subalgebra $\mathfrak{q}$ of $\mathfrak{g l}_{n}\left(\mathrm{H}_{1, \mathbf{c}}(\Gamma)\right)$ with $\mathbf{b}$ the set of its first $n d$ characteristic polynomials, one can define similarly generalized Verma modules $M(\mathfrak{q}, \lambda)$ by choosing $\lambda$ such that $\lambda(h)=0$ for any $h \in \mathfrak{g l}_{n}\left(\mathrm{H}_{1, \mathbf{c}}(\Gamma)\right)[0, \mathbf{b}]$, since, in this case, $\lambda$ descends to $\mathfrak{q} /[\mathfrak{q}, \mathfrak{q}]$; see Proposition 6.8 .

Our goal now is to study quasifinite irreducible highest weight modules, so we introduce the next definition. 
Definition 7.5. We say a graded highest weight module $M=\bigoplus_{k \in \mathbb{Z}} M[k]$ over $\mathfrak{g l}_{n}\left(\mathrm{H}_{1, \mathbf{c}}(\Gamma)\right)$ is quasifinite if $\operatorname{dim}_{\mathbb{C}} M[k]<\infty$ for all $k \in \mathbb{Z}$.

In order to obtain below a condition equivalent to the quasifiniteness of $L(\lambda)$, we need one more definition, as in [Kac and Radul 1993].

Definition 7.6. The Verma module $M(\lambda)$ is said to be highly degenerate if there exists a singular vector $v \in M(\lambda)[-1]$ such that $v=A v_{\lambda}$ with $A \in \mathfrak{g l}_{n}\left(\mathrm{H}_{1, \mathbf{c}}(\Gamma)\right)[-1]$ and $\operatorname{qdet}(A) \neq 0$.

The space $\mathfrak{g l}_{n}\left(\mathrm{H}_{1, \mathbf{c}}(\Gamma)\right)[-1]$ is spanned by $E_{i+1, i} \omega^{r} \mathbf{e}_{l}$ and by $E_{1 n} \omega^{r} u \mathbf{e}_{l}$, so the entries of a matrix $A$ in $\mathfrak{g l}_{n}\left(\mathrm{H}_{1, \mathbf{c}}(\Gamma)\right)[-1]$ do not necessarily belong to a commutative ring. By qdet $(A)$, we thus mean the quasideterminant of $A$ (which, in this case, is, up to a sign, the product of the nonzero entries of $A$ ).

Proposition 7.7. The Verma module $M(\lambda)$ is highly degenerate if and only if $\lambda$ vanishes on $\mathfrak{g l}_{n}\left(\mathrm{H}_{1, \mathbf{c}}(\Gamma)\right)[0, \mathbf{b}]$ for some $n d$ monic (and thus nonzero) polynomials $\mathbf{b}=\left(b^{i, l}(\omega)\right)_{1 \leq i \leq n}^{0 \leq l \leq d-1}$.

Proof. The argument from the proof of [Boyallian et al. 1998, Proposition 4.1] applies. The polynomials $b^{i, l}(\omega)$ are related to the matrix $A$ as follows. Since $A \in \mathfrak{g l}_{n}\left(\mathrm{H}_{t=1, \mathbf{c}}(\Gamma)\right)[-1]$, it can be written as a linear combination of matrices of the type $E_{i+1, i} b^{i+1, l}(w) \mathbf{e}_{l}$ for $1 \leq i \leq n-1$ and $E_{1 n} b^{1, l}(w) u \mathbf{e}_{l}$ with $0 \leq l \leq d-1$. It follows from the proof of Proposition 6.8 that $\mathfrak{g l}_{n}\left(\mathrm{H}_{1, \mathbf{c}}(\Gamma)\right)[0, \mathbf{b}]$ is spanned by $[B, A]$ for all $B \in \mathfrak{g l}_{n}\left(\mathrm{H}_{1, \mathbf{c}}(\Gamma)\right)[1]$.

Proposition 7.8 [Boyallian et al. 1998]. Given $\lambda \in \mathfrak{g l}_{n}\left(\mathrm{H}_{1, \mathbf{c}}(\Gamma)\right)[0]^{*}$ as before, the following conditions are equivalent:

(1) $M(\lambda)$ is highly degenerate.

(2) $L(\lambda)$ is quasifinite.

(3) $L(\lambda)$ is a quotient of a generalized Verma module $M(\mathfrak{q}, \lambda)$ in which all the

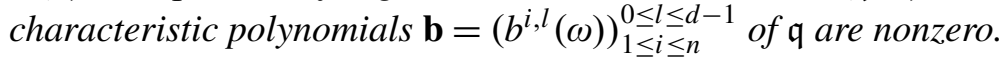

Proof. Proposition 7.7 shows that (1) and (3) are equivalent. Let us show that if all the polynomials $b^{i, l}(\omega)$ are nonzero, then $\operatorname{dim}_{\mathbb{C}}\left(\mathfrak{g l}_{n}\left(\mathrm{H}_{1, \mathbf{c}}(\Gamma)\right)[k] / \mathfrak{q}[k]\right)$ is finite; hence $L(\lambda)$ is quasifinite. Under this assumption, it follows from the proof of Definition 6.6 that $b_{k}^{i, l}(w)$ are nonzero for all $k \in \mathbb{Z}_{\leq-1}, \quad 1 \leq i \leq n, 0 \leq l \leq d-1$. Recall that, for $k<0$, we can write

$$
\mathfrak{g l}_{n}\left(\mathrm{H}_{1, \mathbf{c}}(\Gamma)\right)[k]=\sum_{\substack{s, l, i, j \\-s n+j-i=k}} E_{i j} u^{s} \mathbb{C}[\omega] \mathbf{e}_{l} \quad \text { and } \quad \mathfrak{q}[k]=\sum_{\substack{s, l, i, j \\-s n+j-i=k}} E_{i j} u^{s} \mathbb{C}[\omega] b_{k}^{i, l}(w) \mathbf{e}_{l}
$$

Our claim now follows from the observation that $\operatorname{dim}_{\mathbb{C}}\left(\mathbb{C}[w] /\left(b_{k}^{i, l}(w)\right)\right)<\infty$. 
Now suppose that $L(\lambda)$ is quasifinite. Then $\operatorname{dim}_{\mathbb{C}} L(\lambda)[-1]<\infty$, so, with $\widetilde{M}(\lambda)$ the unique maximal submodule of $M(\lambda)$, we have $\widetilde{M}(\lambda)[-1] \neq\{0\}$. All the vectors in $\tilde{M}(\lambda)[-1] \neq\{0\}$ are singular and at least one satisfies the condition in Definition 7.6. Therefore, $M(\lambda)$ is highly degenerate.

In Theorems 5.2 and 5.4, we stated a criterion in terms of certain power series for the integrability of the simple quotients of Verma modules for $\widehat{\mathfrak{s l}}_{n}(A), \widehat{\mathfrak{s l}}_{n}(B)$ and $\widehat{\mathfrak{s l}}_{n}(C)$. We now want to give a similar criterion for the quasifiniteness of $L(\lambda)$. To achieve this, given $\lambda \in \mathfrak{g l}_{n}\left(\mathrm{H}_{1, \mathbf{c}}(\Gamma)\right)[0]^{*}$ as before, set $d_{i, l, r}=\lambda\left(E_{i i} w^{r} \mathbf{e}_{l}\right)$ for $1 \leq i \leq n$ and $\left.D_{i, l}(z)=\sum_{r=0}^{\infty}\left(d_{i, l, r} / r\right)\right) z^{r}$. Recall that a quasipolynomial is a linear combination of functions of the form $p(z) e^{a z}$, where $p(z)$ is a polynomial and $a \in \mathbb{C}$.

Theorem 7.9. The module $L(\lambda)$ is quasifinite if and only if there exist quasipolynomials $\phi_{i, l}(z)$ for $1 \leq i \leq n$ and $0 \leq l \leq d-1$ such that

$$
\left(1-e^{d z}\right) D_{i, l}(z)= \begin{cases}\phi_{1, l}(z) & \text { if } i=1, \\ \phi_{1, l}(z)+\left(1-e^{d z}\right) \phi_{i, l}(z) & \text { if } 2 \leq i \leq n .\end{cases}
$$

Proof. The proof is similar to the proof of [Boyallian et al. 1998, Theorem 4.1], using the description of $\mathfrak{g l}_{n}\left(\mathrm{H}_{1, \mathbf{c}}(\Gamma)\right)[0, \mathbf{b}]$ given just before Proposition 6.8. Let us explain the differences. Writing $b^{i, l}(\omega)=\omega^{m_{i, l}}+f_{i, l, m_{i, l}-1} \omega^{m_{i, l}-1}+\cdots+f_{i, l, 0}$, we obtain the equations $\sum_{r=0}^{m_{i, l}} f_{i, l, r} F_{i, l, r+\tilde{r}}=0$ for $1 \leq i \leq n$ and $\tilde{r}=0,1, \ldots$, where $F_{i, l, r}=d_{i, l, r}-d_{i-1, l, r}$ for $2 \leq i \leq n$ and $f_{i, l, m_{i, l}}=1$. To express $F_{1, l, r}$ in terms of the $d_{i, l, r}$, we write

$$
\begin{aligned}
& E_{11} u v(\omega+1)^{r} b_{-1}^{1, l}(\omega+1) \mathbf{e}_{l+1}-E_{n n} v u \omega^{r} b_{-1}^{1, l}(\omega) \mathbf{e}_{l} \\
& =-E_{11}(\omega+1)^{r+1} b_{-1}^{1, l}(\omega+1) \mathbf{e}_{l+1}+\left(\tilde{c}_{l}+1\right) E_{11}(\omega+1)^{r} b_{-1}^{1, l}(\omega+1) \mathbf{e}_{l+1} \\
& +E_{n n} \omega^{r+1} b_{-1}^{1, l}(\omega) \mathbf{e}_{l}-\left(\tilde{c}_{l}+1\right) E_{n n} \omega^{r} b_{-1}^{1, l}(\omega) \mathbf{e}_{l}
\end{aligned}
$$

We thus see that

$$
F_{1, l, r}=d_{n, l, r+1}-\left(\tilde{c}_{l}+1\right) d_{n, l, r}-\sum_{j=0}^{r+1}\left(\begin{array}{c}
r+1 \\
j
\end{array}\right) d_{1, l+1, j}+\left(\tilde{c}_{l}+1\right) \sum_{j=0}^{r}\left(\begin{array}{l}
r \\
j
\end{array}\right) d_{1, l+1, j} .
$$

Setting $F_{i, l}(z)=\sum_{r=0}^{\infty} F_{i, l, r} z^{r} / r$ ! for $1 \leq i \leq n$, we conclude as in [Boyallian et al. 1998] that $F_{i, l}(z)$ is a quasipolynomial. For $2 \leq i \leq n$, we can write $F_{i, l}(z)=$ $D_{i, l}(z)-D_{i-1, l}(z)$, and for $i=1$, we have

$$
F_{1, l}(z)=D_{n, l}^{\prime}(z)-\left(\tilde{c}_{l}+1\right) D_{n, l}(z)-\left(e^{z} D_{1, l+1}\right)^{\prime}(z)+\left(\tilde{c}_{l}+1\right) e^{z} D_{1, l+1}(z) .
$$

Here, $D_{i, l}^{\prime}(z)$ is the derivative of $D_{i, l}(z)$. This implies that $D_{i, l}^{\prime}(z)-D_{i-1, l}^{\prime}(z)$ is also a quasipolynomial (for $2 \leq i \leq n$ ), and hence so is

$$
\left(D_{1, l}(z)-e^{z} D_{1, l+1}(z)\right)^{\prime}-\left(\tilde{c}_{l}+1\right)\left(D_{1, l}(z)-e^{z} D_{1, l+1}(z)\right) .
$$


Hence $e^{z} D_{1, l+1}(z)-D_{1, l}(z)$ is a quasipolynomial; thus so is $\left(1-e^{d z}\right) D_{1, l}(z)$.

It is possible to construct quasifinite representations of $\mathfrak{g l}_{n}\left(\mathrm{H}_{1, \mathbf{c}}(\Gamma)\right)$ as tensor products of certain modules. This is where the embeddings $\varphi_{a}^{[m]}$ come into play. Unfortunately, they are not necessarily irreducible.

First, we need to construct irreducible representations of $\overline{\mathfrak{g l}}_{\infty}\left(R_{m}\right)$ using a standard procedure. An element $\lambda \in \overline{\mathfrak{g l}}_{\infty}\left(R_{m}\right)[0]^{*}$ is determined by $\lambda_{k}^{(j)}=\lambda\left(E_{k k} t^{j}\right)$ for $k \in \mathbb{Z}$ and $j=0, \ldots, m$, which we call its labels, following the terminology in [Boyallian et al. 1998]. Using induction from the subalgebra of upper-triangular matrices and its one-dimensional representation determined by such a $\lambda$, we construct a Verma module for $\overline{\mathfrak{g l}}_{\infty}\left(R_{m}\right)$; this Verma module has a unique irreducible highest weight quotient $L(m, \lambda)$.

The following is [Boyallian et al. 1998, Proposition 4.4].

Proposition 7.10. The irreducible $\overline{\mathfrak{g l}}_{\infty}\left(R_{m}\right)$-module $L(m, \lambda)$ is quasifinite if and only if for each $j=0, \ldots, m$, all but finitely many of the $\lambda_{k}^{(j)}-\lambda_{k+1}^{(j)}$ are zero.

Let $\mathbf{m}=\left(m_{1}, \ldots, m_{N}\right) \in \mathbb{Z}_{\geq 0}^{\oplus N}$ and $\lambda=(\lambda(1), \ldots, \lambda(N))$ with $\lambda(i)$ belonging to $\overline{\mathfrak{g l}}_{\infty}\left(R_{m_{i}}\right)[0]^{*}$ such that $L\left(m_{i}, \lambda(i)\right)$ is quasifinite. We can form the tensor product $L(\mathbf{m}, \lambda)=\bigotimes_{i=1}^{N} L\left(m_{i}, \lambda(i)\right)$, which is an irreducible quasifinite representation of $\overline{\mathfrak{g l}}_{\infty}[\mathbf{m}]=\bigoplus_{i=1}^{N} \overline{\mathfrak{g l}}_{\infty}\left(R_{m_{i}}\right)$. By pulling it back via the map

$$
\varphi_{\mathbf{a}}^{[\mathbf{m}]}=\bigoplus_{i=1}^{N} \varphi_{a_{i}}^{\left[m_{i}\right]}: \mathfrak{g l}_{n}\left(\mathrm{H}_{t=1, \mathbf{c}}(\Gamma)\right) \rightarrow \overline{\mathfrak{g l}}_{\infty}[\mathbf{m}] \quad \text { for } \mathbf{a}=\left(a_{1}, \ldots, a_{N}\right) \in \mathbb{C}^{N},
$$

we obtain a representation of $\mathfrak{g l}_{n}\left(\mathrm{H}_{t=1, \mathbf{c}}(\Gamma)\right)$, which we denote by $\mathrm{L}_{\mathbf{a}}(\mathbf{m}, \lambda)$.

[Boyallian et al. 1998, Theorem 4.2] does not hold for $\mathfrak{g l}_{n}\left(\mathrm{H}_{t=1, \mathbf{c}}(\Gamma)\right)$, so we cannot deduce that the representation $\mathrm{L}_{\mathbf{a}}(\mathbf{m}, \lambda)$ is necessarily irreducible. Let us discuss what is the difference here. That theorem states that pulling back a quasifinite representation of $\overline{\mathfrak{g l}}_{\infty}[\mathbf{m}]$ to $\mathfrak{g l}_{n}\left(\mathbf{A}_{1} \rtimes \Gamma\right)$ via $\varphi_{\mathbf{a}}^{[\mathbf{m}]}$ gives a representation that has the same submodules. (The proof in the case $\Gamma=\{1\}$ extends to any $d>1$.) The main ideas of the proof are as follows; see also [Kac and Radul 1993]. First, we have to introduce a holomorphic enlargement of $\mathfrak{g l}_{n}\left(\mathbf{A}_{1} \rtimes \Gamma\right)$, as at the end of Section 6: It is the Lie algebra $\mathfrak{g l}_{n}\left(\mathbf{A}_{1}^{\mathbb{O}} \rtimes \Gamma\right)$ spanned by $E_{i j} u^{s} f(\omega) \mathbf{e}_{l}$, where $f(\omega)$ is an entire function of $\omega$, the bracket of $\mathfrak{g l}_{n}\left(\mathbf{A}_{1} \rtimes \Gamma\right)$ extending to $\mathfrak{g l}_{n}\left(\mathbf{A}_{1}^{\mathbb{O}} \rtimes \Gamma\right)$ naturally. Second, the formula for the embedding $\varphi_{\mathbf{a}}^{[\mathbf{m}]}$ (when $a_{i} \neq a_{j}$ for $i \neq j$ ) can be used to obtain a map $\varphi_{\mathbf{a}}^{[\mathbf{m}], \mathcal{O}}: \mathfrak{g l}_{n}\left(\mathbf{A}_{1}^{\mathbb{O}} \rtimes \Gamma\right) \rightarrow \overline{\mathfrak{g l}}_{\infty}[\mathbf{m}]$, which is onto, but not necessarily into. The last step is to show that, if $V$ is a quasifinite module over $\mathfrak{g l}_{n}\left(\mathbf{A}_{1} \rtimes \Gamma\right)$, then, by continuity, we can make $\mathfrak{g l}_{n}\left(\mathbf{A}_{1}^{0} \rtimes \Gamma\right)[k]$ act on $V$ if $k \neq 0$. This involves computing an upper bound on the norm of certain operators.

The first and third step work also for $\mathrm{H}_{t=1, \mathrm{c}}(\Gamma)$, but the second doesn't. Consider the algebra $\mathrm{H}_{t=1, \mathbf{c}}^{\mathbb{C}}(\Gamma)$ spanned by elements of the form $v^{r} f(\omega) \mathbf{e}_{l}$ and $u^{s} f(\omega) \mathbf{e}_{l}$, 
where $f(\omega)$ is an entire function of $\omega$ and the multiplication is given by (in the case $r \geq s$ )

$v^{r} f(\omega) \mathbf{e}_{l_{1}} u^{s} g(\omega) \mathbf{e}_{l_{2}}=\delta_{l_{1}-s, l_{2}} v^{r-s}\left(\prod_{k=1}^{s}\left(-\omega+c_{l_{2}+s-k}+1+s-k\right)\right) f(\omega-s) g(\omega) \mathbf{e}_{l_{2}}$.

We have also a map $\varphi_{\mathbf{a}}^{[\mathbf{m}], \mathscr{O}}: \mathfrak{g l}_{n}\left(\mathrm{H}_{t=1, \mathbf{c}}^{\mathcal{C}}(\Gamma)\right) \rightarrow \overline{\mathfrak{g l}}_{\infty}[\mathbf{m}]$, but it is not onto; for instance, if $a=0=m=c_{l}$ for $l=0, \ldots, d-1$, then $\varphi_{a}^{[m]}\left(E_{i j} v f(\omega) \mathbf{e}_{k}\right)=$ $\sum_{l \in \mathbb{Z}}(l d+k) f(-l d-k) E_{(l d+k-1) n+i-1,(l d+k) n+j-1}$. Therefore, in the image, the coefficient of $E_{-n+i-1, j-1}$ is always zero, independently of $f(\omega)$. At least, we have the following result.

Proposition 7.11. Assume that $a_{i}-a_{j} \notin \mathbb{Z}$ for $1 \leq i \neq j \leq N$ and $\tilde{c}_{k}+a_{i} \notin \mathbb{Z}$ for all $1 \leq i \leq N$ and $0 \leq k \leq d-1$. Then $\varphi_{\mathbf{a}}^{[\mathbf{m}], \mathcal{O}}: \mathfrak{g l}_{n}\left(\mathrm{H}_{t=1, \mathbf{c}}^{\mathcal{O}}(\Gamma)\right) \rightarrow \overline{\mathfrak{g l}}_{\infty}[\mathbf{m}]$ is onto.

Proof. Decompose $\overline{\mathfrak{g l}}_{\infty}$ as $\overline{\mathfrak{g l}}_{\infty}=\overline{\mathfrak{n}}_{\infty}^{-} \oplus \overline{\mathfrak{h}}_{\infty} \oplus \overline{\mathfrak{n}}_{\infty}^{+}$, where $\overline{\mathfrak{h}}_{\infty}$ is the Lie subalgebra of all the diagonal blocks of size $n$ (with one having a corner at the $(0,0)$-entry), and $\overline{\mathfrak{n}}_{\infty}^{ \pm}$are the complements of $\overline{\mathfrak{h}}_{\infty}$ consisting of strictly upper and lower triangular matrices. That $\varphi_{\mathbf{a}}^{[\mathbf{m}], \mathcal{O}}$ is onto the subspace $\overline{\mathfrak{n}}_{\infty}^{-}$when restricted to the subspace spanned by the elements $E_{i j} u^{s} f(\omega) \mathbf{e}_{k}$ with $s \in \mathbb{Z}_{\geq 0}, 1 \leq i, j \leq n$ and $0 \leq k \leq d-1$ follows from [Boyallian et al. 1998; Kac and Radul 1993], so let us focus instead on $\mathfrak{g l}_{n}\left(\mathrm{H}_{t=1, \mathbf{c}}^{0}(\Gamma)\right)[>0]$. Explicitly, using the Taylor formula for the expansion of a function of $t$ around $t=0$ and (7), $\varphi_{a_{i}}^{\left[m_{i}\right]}$ is given by

$$
\varphi_{a_{i}}^{\left[m_{i}\right]}\left(E_{i j} v^{s} f(\omega) \mathbf{e}_{k}\right)=\sum_{l \in \mathbb{Z}} \sum_{b=0}^{m_{i}} \frac{g^{(b)}\left(a_{i}+l d\right)}{b !} t^{b} E_{(l d+k-s) n+i-1,(l d+k) n+j-1}
$$

if we set $g(t)=\left(\prod_{p=0}^{s-1}\left(k-p+t+\tilde{c}_{k-p-1}\right)\right) f(-k-t)$. As in [Kac and Radul 1993, Proposition 3.1], we can use the fact that, for every discrete set of points in $\mathbb{C}$, there is a holomorphic function on $\mathbb{C}$ with prescribed values of its first $m_{i}$ derivatives at each points of such a set. Combining this with our assumption that $a_{i}-a_{j} \notin \mathbb{Z}$ for $1 \leq i \neq j \leq N$, we deduce that, given a matrix $E=\bigoplus_{i=1}^{N} E_{i}$ in $\overline{\mathfrak{g l}}_{\infty}[\mathbf{m}]$, there exists an entire function $g(t)$ such that the right side of (8) is equal to $E_{i}$ for all $i=1, \ldots, N$. To complete the proof, we have to find an entire function $f(\omega)$ such that, if we set $\tilde{g}(t)=\left(\prod_{p=0}^{s-1}\left(k-p+t+\tilde{c}_{k-p-1}\right)\right) f(-k-t)$, then

$$
g^{(b)}\left(a_{i}+l d\right)=\tilde{g}^{(b)}\left(a_{i}+l d\right) \quad \text { for } 1 \leq i \leq N, 0 \leq b \leq m_{i} \text { and all } l \in \mathbb{Z} .
$$

Set $P(t)=\prod_{p=0}^{s-1}\left(k-p+t+\tilde{c}_{k-p-1}\right)$, so that

$$
\tilde{g}^{(b)}(t)=\sum_{a=0}^{b}\left(\begin{array}{l}
b \\
a
\end{array}\right) P^{(n-a)}(t) f^{(a)}(-k-t) .
$$


Fix $1 \leq i \leq N$ and $l \in \mathbb{Z}$ and consider the system of equations

$$
g^{(b)}\left(a_{i}+l d\right)=\sum_{a=0}^{b}\left(\begin{array}{l}
b \\
a
\end{array}\right) P^{(b-a)}\left(a_{i}+l d\right) z_{a} \text { for } b=0,1, \ldots, m_{i},
$$

with $z_{0}, \ldots, z_{m_{i}}$ being the unknown variables (which we would like to express in terms of $\left.\tilde{g}^{(b)}\left(a_{i}+l d\right)\right)$. Our hypothesis that $\tilde{c}_{k}+a_{i} \notin \mathbb{Z}$ implies that $P\left(a_{i}+l d\right) \neq 0$, so the matrix of this system is triangular with nonzero entries along the diagonal. We can thus solve it: Let $\tilde{z}_{i, l}^{0}, \tilde{z}_{i, l}^{1}, \ldots, \tilde{z}_{i, l}^{m_{i}}$ be a solution. Then we can rephrase the problem by saying that we now have to find an entire function $f(\omega)$ such that $f^{(a)}\left(a_{i}+l d\right)=\tilde{z}_{i, l}^{a}$ for $1 \leq i \leq N, 0 \leq a \leq m_{i}$ and all $l \in \mathbb{Z}$. To deduce the existence of such a function, we can now apply the same argument as the one used to deduce the existence of $g(\omega)$ above.

The representation $L_{\mathbf{a}}(\mathbf{m}, \lambda)$ is a highest weight module, so it is interesting to calculate its associated series $\mathbf{D}_{i, k}(z)$, which is equal to $\sum_{j=1}^{N} D_{i, j, k}(z)$. The formulas are similar to those in [Boyallian et al. 1998]. Set

$$
h_{l}^{(p)}(j)=\lambda_{l}^{(p)}(j)-\lambda_{l+1}^{(p)}(j) \quad \text { and } \quad g_{j, l}(z)=\sum_{p=0}^{m_{j}} h_{l}^{(p)}(j)(-z)^{p} / p ! .
$$

We have

$$
\begin{aligned}
& D_{i, j, k}(z)= \sum_{p=1}^{m_{j}} \sum_{l \in \mathbb{Z}} \lambda_{(l d+k) n+i-1}^{(p)}(j) \frac{(-z)^{p}}{p !} e^{-\left(a_{j}+l d+k\right) z} \\
& D_{i, j, k}(z)=\left(1-e^{d z}\right)^{-1} \sum_{l \in \mathbb{Z}} e^{-\left(a_{j}+l d+k\right) z}\left(g_{j,(l d+k) n+i-1}(z)+g_{j,(l d+k) n+i}(z)+\cdots\right. \\
&\left.+g_{j,(l d+k) n+d n+i-2}(z)\right)
\end{aligned}
$$

\section{Further discussions}

We now present further possible research directions related to the results herein.

8A. Double affine Lie algebras and Kleinian singularities. Let $G$ be an arbitrary finite subgroup of $\mathrm{SL}_{2}(\mathbb{C})$. Such a group $G$ does not always act on the torus $\mathbb{C}^{\times 2}$ or on $\mathbb{C} \times \mathbb{C}^{\times}$, so we can consider only the algebras $\mathbb{C}[u, v] \rtimes G$ and $\mathbb{C}[u, v]^{G}$. Moreover, when $G$ is not cyclic, each of these Lie algebras has only one triangular decomposition, namely

$$
\begin{aligned}
\mathfrak{s l}_{n}(\mathbb{C}[u, v] \rtimes G) & \cong \mathfrak{n}^{-}(\mathbb{C}[u, v] \rtimes G) \oplus \mathfrak{h}(\mathbb{C}[u, v] \rtimes G) \oplus \mathfrak{n}^{+}(\mathbb{C}[u, v] \rtimes G), \\
\mathfrak{s l}_{n}\left(\mathbb{C}[u, v]^{G}\right) & \cong \mathfrak{n}^{-}\left(\mathbb{C}[u, v]^{G}\right) \oplus \mathfrak{h}(\mathbb{C}[u, v] \rtimes G) \oplus \mathfrak{n}^{+}\left(\mathbb{C}[u, v]^{G}\right)
\end{aligned}
$$

These also admit universal central extensions. Since $\mathbb{C}[u, v]^{G}$ is commutative, $H C_{1}\left(\mathbb{C}[u, v]^{G}\right)=\Omega^{1}\left(\mathbb{C}[u, v]^{G}\right) / d\left(\mathbb{C}[u, v]^{G}\right)$. We know from [Kassel 1984] that 
the bracket on the universal central extension

$$
\widehat{\mathfrak{s l}}_{n}\left(\mathbb{C}[u, v]^{G}\right)=\mathfrak{s l}_{n}\left(\mathbb{C}[u, v]^{G}\right) \oplus\left(\Omega^{1}\left(\mathbb{C}[u, v]^{G}\right) / d\left(\mathbb{C}[u, v]^{G}\right)\right)
$$

of $\mathfrak{s l}_{n}\left(\mathbb{C}[u, v]^{G}\right)$ is given by

$$
\left[m_{1} \otimes p_{1}, m_{2} \otimes p_{2}\right]=\left[m_{1}, m_{2}\right] \otimes\left(p_{1} p_{2}\right)+\operatorname{Tr}\left(m_{1} m_{2}\right) p_{1} d p_{2} .
$$

As for $\widehat{\mathfrak{s l}}_{n}(\mathbb{C}[u, v] \rtimes G)$, it is known that its kernel $H C_{1}(\mathbb{C}[u, v] \rtimes G)$ is equal to $\Omega^{1}(\mathbb{C}[u, v])^{G} / d\left(\mathbb{C}[u, v]^{G}\right)$; see [Farinati 2005]. However, to obtain an explicit formula for its bracket, one would have to choose a splitting

$$
\langle\mathbb{C}[u, v] \rtimes G, \mathbb{C}[u, v] \rtimes G\rangle=[\mathbb{C}[u, v] \rtimes G, \mathbb{C}[u, v] \rtimes G] \oplus H C_{1}(\mathbb{C}[u, v] \rtimes G) ;
$$

see Section 2.

In [Kapranov and Vasserot 2000], the authors proved that the derived category of coherent sheaves on the minimal resolution $\overline{\mathbb{C}^{2} / G}$ of the singularity $\mathbb{C}^{2} / G$ is equivalent to the derived category of modules over the skew-group ring $\mathbb{C}[u, v] \rtimes G$. It is thus natural to ask if there is a connection between the derived category of representations of $\mathfrak{s l}_{n}(\mathbb{C}[u, v] \rtimes G)$ and the derived category of modules over a certain sheaf of Lie algebras on $\overline{\mathbb{C}^{2} / G}$.

In the same line of thought, since $A_{1} \rtimes G$ and $A_{1}^{G}$ are Morita equivalent, one can wonder about the connections between $\mathfrak{s l}_{n}\left(A_{1} \rtimes G\right)$ and $\mathfrak{s l}_{n}\left(A_{1}^{G}\right)$. However, even if $A$ and $B$ are Morita equivalent rings, the categories of representations of $\mathfrak{s l}_{n}(A)$ and $\mathfrak{s l}_{n}(B)$ are not necessarily equivalent. As a counterexample, one can consider $A=\mathbb{C}\left[t^{ \pm 1}\right]$ and $B=\mathbb{C}\left[u^{ \pm 1}\right] \rtimes(\mathbb{Z} / d \mathbb{Z}) \cong M_{d}\left(\mathbb{C}\left[t^{ \pm 1}\right]\right)$, in which case $\mathfrak{s l}_{n}(B)=\mathfrak{s l}_{n d}\left(\mathbb{C}\left[t^{ \pm 1}\right]\right)$.

The definitions of Weyl modules recalled in Section 5B can be adapted to both $\mathfrak{s l}_{n}(\mathbb{C}[u, v] \rtimes G)$ and $\mathfrak{s l}_{n}\left(\mathbb{C}[u, v]^{G}\right)$. Studying these appears to be a reasonable way to approach the representation theory of these Lie algebras since, when $G$ is not cyclic, we do not have triangular decompositions similar to (3) or presentations as in Proposition 5.3. It would be interesting to compute the dimension of local Weyl modules at the Kleinian singularity. For smooth points on an affine variety and certain highest weights, the dimension of local Weyl modules was computed in [Feigin and Loktev 2004], and [Kuwabara 2006] treated the case of a double point. One can expect the study of such local Weyl modules to be related to the geometry of the minimal resolution of the Kleinian singularity.

8B. Quiver Lie algebras. Symplectic reflection algebras for wreath products of $G$ are known to be Morita equivalent to certain deformed preprojective algebras of affine Dynkin quivers, which are called Gan-Ginzburg algebras in the literature [Gan and Ginzburg 2005]. In the rank one case, these are the usual deformed preprojective algebras $\Pi^{\lambda}(Q)$. The affine Dynkin diagram in question is associated 
to $G$ via the McKay correspondence. The quantum Lie algebra analogues of these Gan-Ginzburg algebras were introduced in [Guay 2009a] and are deformations of the enveloping algebra of a Lie algebra which is slightly larger than the universal central extension of $\mathfrak{s l}_{n}(\Pi(Q))$, where $\Pi(Q)=\Pi^{\lambda=0}(Q)$. The same themes as in the previous sections can be studied in the context of the Lie algebra $\mathfrak{s l}_{n}(\Pi(Q))$, in particular when the graph underlying $Q$ is an affine Dynkin diagram. Actually, when $Q$ is the cyclic quiver on $d$ vertices, $\Pi(Q) \cong \mathbb{C}[u, v] \rtimes \Gamma$. Furthermore, if $e_{0}$ is the extending vertex of an affine Dynkin diagram, then $e_{0} \Pi(Q) e_{0} \cong \mathbb{C}[u, v]^{G}$.

All these are examples of matrix Lie algebras over interesting noncommutative rings. It is possible to replace $\mathfrak{s l}_{n}$ by another semisimple Lie algebra. This is explained in [Berenstein and Retakh 2008]. It would also be interesting to compare our work with the constructions in [Halbout et al. 2008].

\section{Acknowledgments}

Guay gratefully acknowledges the hospitality of the Laboratoire de Mathématiques de l'Université de Versailles-St-Quentin-en-Yvelines where this project was started while he was a postdoctoral researcher supported by the Ministère français de la Recherche. He is also grateful for the support received from the University of Edinburgh, the University of Alberta and an NSERC Discovery Grant. Loktev was partially supported by RF President Grant N.Sh-3035.2008.2, grants RFBR08-02-00287, RFBR-CNRS-07-01-92214 and RFBR-IND-0801-91300, and the P. Deligne 2004 Balzan prize in mathematics. We thank A. Pianzola for pointing out the reference [Berman et al. 2003]. We are grateful to I. Gordon for his comments, for his suggestions regarding Corollary 5.21 and for pointing out the counterexample from the work of M. Haiman that we presented at the end of Section 5C.

\section{References}

[Alev et al. 2000] J. Alev, M. A. Farinati, T. Lambre, and A. L. Solotar, "Homologie des invariants d'une algèbre de Weyl sous l'action d'un groupe fini”, J. Algebra 232:2 (2000), 564-577. MR 2002c:16047 Zbl 1002.16005

[Allison et al. 1997] B. N. Allison, S. Azam, S. Berman, Y. Gao, and A. Pianzola, Extended affine Lie algebras and their root systems, Mem. Amer. Math. Soc. 126:603, Amer. Math. Soc., Providence, RI, 1997. MR 97i:17015 Zbl 0879.17012

[Berenstein and Retakh 2008] A. Berenstein and V. Retakh, "Lie algebras and Lie groups over noncommutative rings", Adv. Math. 218:6 (2008), 1723-1758. MR 2009d:17008 Zbl 05312130

[Berman et al. 2003] S. Berman, Y. Gao, and S. Tan, "A unified view of some vertex operator constructions", Israel J. Math. 134 (2003), 29-60. MR 2004c:17057 Zbl 1035.17038

[Boyallian and Liberati 2002] C. Boyallian and J. I. Liberati, "On modules over matrix quantum pseudo-differential operators”, Lett. Math. Phys. 60:1 (2002), 73-85. MR 2003b:17028 Zbl 1009. 17017 
[Boyallian et al. 1998] C. Boyallian, V. G. Kac, J. I. Liberati, and C. H. Yan, "Quasifinite highest weight modules over the Lie algebra of matrix differential operators on the circle", J. Math. Phys. 39:5 (1998), 2910-2928. MR 99c:17012 Zbl 0999.17032

[Chari 1986] V. Chari, "Integrable representations of affine Lie-algebras", Invent. Math. 85:2 (1986), 317-335. MR 88a:17034 Zbl 0603.17011

[Chari and Le 2003] V. Chari and T. Le, "Representations of double affine Lie algebras", pp. 199219 in A tribute to C. S. Seshadri (Chennai, 2002), edited by V. Lakshmibai et al., Birkhäuser, Basel, 2003. MR 2004k:17044 Zbl 1107.17012

[Chari and Loktev 2006] V. Chari and S. Loktev, "Weyl, Demazure and fusion modules for the current algebra of $\mathfrak{s l}_{r+1}$ ", Adv. Math. 207:2 (2006), 928-960. MR 2008a:17029 Zbl 1161.17318

[Chari and Pressley 2001] V. Chari and A. Pressley, "Weyl modules for classical and quantum affine algebras", Represent. Theory 5 (2001), 191-223. MR 2002g:17027 Zbl 0989.17019

[Crawley-Boevey 1991] W. W. Crawley-Boevey, "Regular modules for tame hereditary algebras", Proc. London Math. Soc. (3) 62:3 (1991), 490-508. MR 92b:16024 Zbl 0768.16003

[Etingof and Ginzburg 2002] P. Etingof and V. Ginzburg, "Symplectic reflection algebras, CalogeroMoser space, and deformed Harish-Chandra homomorphism", Invent. Math. 147:2 (2002), 243348. MR 2003b:16021 Zbl 1061.16032

[Farinati 2005] M. Farinati, "Hochschild duality, localization, and smash products", J. Algebra 284:1 (2005), 415-434. MR 2005j:16009 Zbl 1066.16010

[Feigin and Loktev 2004] B. Feigin and S. Loktev, "Multi-dimensional Weyl modules and symmetric functions", Comm. Math. Phys. 251:3 (2004), 427-445. MR 2005m:17005 Zbl 1100.17005

[Gan and Ginzburg 2005] W. L. Gan and V. Ginzburg, "Deformed preprojective algebras and symplectic reflection algebras for wreath products", J. Algebra 283:1 (2005), 350-363. MR 2005h: 16049 Zbl 1133.16013

[Garland 1978] H. Garland, “The arithmetic theory of loop algebras”, J. Algebra 53:2 (1978), 480551. MR 80a:17012 Zbl 0383.17012

[Ginzburg et al. 1995] V. Ginzburg, M. Kapranov, and É. Vasserot, "Langlands reciprocity for algebraic surfaces”, Math. Res. Lett. 2:2 (1995), 147-160. MR 96f:11086 Zbl 0914.11040

[Ginzburg et al. 2003] V. Ginzburg, N. Guay, E. Opdam, and R. Rouquier, "On the category 0 for rational Cherednik algebras”, Invent. Math. 154:3 (2003), 617-651. MR 2005f:20010 Zbl 1071. 20005

[Gordon 2003] I. Gordon, "On the quotient ring by diagonal invariants", Invent. Math. 153:3 (2003), 503-518. MR 2004f:20075 Zbl 1039.20019

[Gordon 2007] I. Gordon, "Gelfand-Kirillov conjecture for symplectic reflection algebras", preprint, 2007. arXiv 0710.1419

[Guay 2005] N. Guay, "Cherednik algebras and Yangians", Int. Math. Res. Not. 2005:57 (2005), 3551-3593. MR 2006m:16040 Zbl 1096.20006

[Guay 2007] N. Guay, "Affine Yangians and deformed double current algebras in type A", Adv. Math. 211:2 (2007), 436-484. MR 2008d:17020 Zbl 1142.17008

[Guay 2009a] N. Guay, "Quantum algebras and quivers", Selecta Math. (N.S.) 14:3-4 (2009), 667700.

[Guay 2009b] N. Guay, "Quantum algebras and symplectic reflection algebras for wreath products", preprint, 2009, Available at http://www.math.ualberta.ca/ nguay/NGuayGammaDDCA.pdf.

[Haiman 1994] M. D. Haiman, "Conjectures on the quotient ring by diagonal invariants", J. Algebraic Combin. 3:1 (1994), 17-76. MR 95a:20014 Zbl 0803.13010 
[Haiman 2002] M. Haiman, "Vanishing theorems and character formulas for the Hilbert scheme of points in the plane", Invent. Math. 149:2 (2002), 371-407. MR 2003f:14006 Zbl 1053.14005

[Halbout et al. 2008] G. Halbout, J.-M. Oudom, and X. Tang, "Deformations of linear Poisson orbifolds", preprint, 2008. arXiv 0807.0027

[Hernandez 2005] D. Hernandez, "Representations of quantum affinizations and fusion product", Transform. Groups 10:2 (2005), 163-200. MR 2006k:17025 Zbl 1102.17009

[Hernandez 2007] D. Hernandez, "Drinfeld coproduct, quantum fusion tensor category and applications”, Proc. Lond. Math. Soc. (3) 95:3 (2007), 567-608. MR 2008k:17017 Zbl 1133.17010

[Hernandez 2009] D. Hernandez, "Quantum toroidal algebras and their representations", Selecta Math. (N.S.) 14:3-4 (2009), 701-725.

[Kac 1968] V. G. Kac, "Simple irreducible graded Lie algebras of finite growth", Izv. Akad. Nauk SSSR Ser. Mat. 32 (1968), 1323-1367. MR 41 \#4590 Zbl 0222.17007

[Kac 1990] V. G. Kac, Infinite-dimensional Lie algebras, 3rd ed., Cambridge University Press, 1990. MR 92k:17038 Zbl 0716.17022

[Kac and Radul 1993] V. Kac and A. Radul, "Quasifinite highest weight modules over the Lie algebra of differential operators on the circle", Comm. Math. Phys. 157:3 (1993), 429-457. MR 95f: 81036 Zbl 0826.17027

[Kapranov and Vasserot 2000] M. Kapranov and E. Vasserot, "Kleinian singularities, derived categories and Hall algebras”, Math. Ann. 316:3 (2000), 565-576. MR 2001h:14012 Zbl 0997.14001

[Kassel 1984] C. Kassel, "Kähler differentials and coverings of complex simple Lie algebras extended over a commutative algebra", pp. 265-275 in Proceedings of the Luminy conference on algebraic K-theory (Luminy, 1983), edited by E. M. Friedlander and M. Karoubi, J. Pure Appl. Algebra 34:2-3, 1984. MR 86h:17013

[Kassel and Loday 1982] C. Kassel and J.-L. Loday, "Extensions centrales d'algèbres de Lie", Ann. Inst. Fourier (Grenoble) 32:4 (1982), 119-142 (1983). MR 85g:17004 Zbl 0485.17006

[Khare 2009] A. Khare, "Functoriality of the BGG category O", preprint, 2009. To appear in Comm. Alg. arXiv 0811.2073v1

[Kuwabara 2006] T. Kuwabara, "Symmetric coinvariant algebras and local Weyl modules at a double point”, J. Algebra 295:2 (2006), 426-440. MR 2006i:13010 Zbl 1108.13006

[Loktev 2008] S. Loktev, "Weight multiplicity polynomials of multi-variable Weyl modules", preprint, 2008. arXiv 0806.0170

[Mathieu 1986] O. Mathieu, "Classification des algèbres de Lie graduées simples de croissance $\leq 1$ ", Invent. Math. 86:2 (1986), 371-426. MR 88a:17008 Zbl 0615.17010

[Mathieu 1992] O. Mathieu, "Classification of simple graded Lie algebras of finite growth", Invent. Math. 108:3 (1992), 455-519. MR 93h:17069 Zbl 0769.17018

[Miki 2000] K. Miki, "Representations of quantum toroidal algebra $U_{q}\left(\mathrm{sl}_{n+1, \text { tor }}\right)(n \geq 2)$ ", J. Math. Phys. 41:10 (2000), 7079-7098. MR 2001j:17033 Zbl 1028.17011

[Miki 2004] K. Miki, "Integrable irreducible highest weight modules for $\operatorname{sl}_{2}\left(\mathbf{C}_{p}\left[x^{ \pm 1}, y^{ \pm 1}\right]\right)$ ", Osaka J. Math. 41:2 (2004), 295-326. MR 2005d:17024 Zbl 1138.17308

[Moody et al. 1990] R. V. Moody, S. E. Rao, and T. Yokonuma, “Toroidal Lie algebras and vertex representations”, Geom. Dedicata 35:1-3 (1990), 283-307. MR 91i:17032 Zbl 0704.17011

[Nagao 2007] K. Nagao, "K-theory of quiver varieties, q-Fock space and nonsymmetric Macdonald polynomials", preprint, 2007. arXiv 0709.1767

[Nakajima 2001] H. Nakajima, "Quiver varieties and finite-dimensional representations of quantum affine algebras”, J. Amer. Math. Soc. 14:1 (2001), 145-238. MR 2002i:17023 Zbl 0981.17016 
[Nakajima 2002] H. Nakajima, "Geometric construction of representations of affine algebras", pp. 423-438 in Proceedings of the International Congress of Mathematicians, I (Beijing, 2002), edited by T. Li, Higher Ed. Press, Beijing, 2002. MR 2004e:17012 Zbl 1049.17014

[Rao 2004] S. E. Rao, "On representations of toroidal Lie algebras", pp. 146-167 in Functional analysis VIII, edited by D. Bakić et al., Various Publ. Ser. 47, Århus Univ., 2004. MR 2006e:17033 Zbl 1146.17311

[Schiffmann 2006] O. Schiffmann, "Canonical bases and moduli spaces of sheaves on curves", Invent. Math. 165:3 (2006), 453-524. MR 2008b:17023 Zbl 1142.17004

[Shoikhet 1998] B. B. Shoikhet, "Certain topics on the representation theory of the Lie algebra gl $(\lambda)$ ", pp. 3764-3806 in Complex analysis and representation theory, I, edited by V. P. Saharova, J. Math. Sci. (New York) 92:2, 1998. MR 2000m:17005 Zbl 0916.17019

[Vale 2007] R. Vale, "Rational Cherednik algebras and diagonal coinvariants of $G(m, p, n)$ ", $J$. Algebra 311:1 (2007), 231-250. MR 2008c:20077 Zbl 1116.13005

[Varagnolo and Vasserot 1996] M. Varagnolo and E. Vasserot, "Schur duality in the toroidal setting", Comm. Math. Phys. 182:2 (1996), 469-483. MR 98a:17024 Zbl 0879.17007

[Varagnolo and Vasserot 1998] M. Varagnolo and E. Vasserot, "Double-loop algebras and the Fock space”, Invent. Math. 133:1 (1998), 133-159. MR 99g:17035 Zbl 0904.17014

[Yoon 2002] Y. Yoon, "On the polynomial representations of current algebras", J. Algebra 252:2 (2002), 376-393. MR 2003g:17010 Zbl 1031.17006

Received January 6, 2009. Revised June 13, 2009.

NiCOLAS GUAY

DEPARTMENT OF MATHEMATICAL AND Statistical SCIENCES

UNIVERSITY OF ALBERTA

CAB 632

Edmonton, Alberta T6G 2G1

CANADA

nguay@math.ualberta.ca

DAVID HERNANDEZ

CNRS - ÉCOLE NORMALE SUPÉRIEURE

45, RUE D'ULM

75005 PARIS

FRANCE

David.Hernandez@ens.fr

\section{SERGEY LOKTEV}

INSTITUTE FOR THEORETICAL AND EXPERIMENTAL PHYSICS

Moscow 117218

RUSSIA

loktev@itep.ru 